\title{
Neonatal Injury Evokes Persistent Deficits in Dynorphin Inhibitory Circuits within the Adult Mouse Superficial Dorsal Horn
}

\author{
Chelsie L. Brewer, ${ }^{1,2^{*}}{ }^{\oplus}$ Jie Li, ${ }^{2 *}$ Keith 0'Conor, ${ }^{3}{ }^{\circledR}$ Elizabeth K. Serafin, ${ }^{2}$ and ${ }^{\circledR}$ Mark L. Baccei ${ }^{1,2}$ \\ ${ }^{1}$ Neuroscience Graduate Program, University of Cincinnati College of Medicine, Cincinnati, Ohio 45267, ${ }^{2}$ Pain Research Center, Department of \\ Anesthesiology, University of Cincinnati College of Medicine, Cincinnati, Ohio 45267, and ${ }^{3}$ Medical Student Summer Research Program, University \\ of Cincinnati College of Medicine, Cincinnati, Ohio 45267
}

Neonatal tissue damage induces long-term deficits in inhibitory synaptic transmission within the spinal superficial dorsal horn (SDH) that include a reduction in primary afferent-evoked, feedforward inhibition onto adult projection neurons. However, the subpopulations of mature GABAergic interneurons which are compromised by early-life injury have yet to be identified. The present research illuminates the persistent effects of neonatal surgical injury on the function of inhibitory SDH interneurons derived from the prodynorphin (DYN) lineage, a population that synapses directly onto lamina I spinoparabrachial neurons and is known to suppress mechanical pain and itch in adults. The results demonstrate that hindpaw incision at postnatal day 3 (P3) significantly decreased the strength of primary afferent-evoked glutamatergic drive onto DYN neurons within the adult mouse SDH while increasing the appearance of afferent-evoked inhibition onto the same population. Neonatal injury also dampened the intrinsic membrane excitability of mature DYN neurons, and reduced their action potential discharge in response to sensory input, compared with naive littermate controls. Furthermore, P3 incision decreased the efficacy of inhibitory DYN synapses onto adult spinoparabrachial neurons, which reflected a prolonged reduction in the probability of GABA release. Collectively, the data suggest that early-life tissue damage may persistently constrain the ability of spinal DYN interneurons to limit ascending nociceptive transmission to the adult brain. This is predicted to contribute to the loss of feedforward inhibition onto mature projection neurons, and the "priming" of nociceptive circuits in the developing spinal cord, following injuries during the neonatal period.

Key words: dorsal horn; GABA; interneuron; patch clamp; spinal cord; spinoparabrachial neuron

Significance Statement

Neonatal injury has lasting effects on pain processing in the adult CNS, including a reduction in feedforward inhibition onto ascending projection neurons in the spinal dorsal horn. While it is clear that spinal GABAergic interneurons are comprised of multiple subpopulations that play distinct roles in somatosensation, the identity of those interneurons which are compromised by tissue damage during early life remains unknown. Here we document persistent deficits in spinal inhibitory circuits involving dynorphin-lineage interneurons previously implicated in gating mechanical pain and itch. Notably, neonatal injury reduced the strength of dynorphin-lineage inhibitory synapses onto mature lamina I spinoparabrachial neurons, a major output of the spinal nociceptive network, which could contribute to the priming of pain pathways by early tissue damage.

Received Jan. 6, 2020; revised Mar. 18, 2020; accepted Apr. 4, 2020.

Author contributions: C.L.B., J.L., E.K.S., and M.L.B. designed research; C.L.B., J.L., K.O., and E.K.S. performed research; C.L.B., J.L., K.O., and E.K.S. analyzed data; C.L.B. wrote the first draft of the paper; C.L.B.,

J.L., E.K.S., and M.L.B. edited the paper; C.L.B., E.K.S., and M.L.B. wrote the paper.

${ }^{*}$ C.L.B. and J.L. contributed equally to this work.

The authors declare no competing financial interests.

This work was supported by National Institutes of Health Grant NS080889 to M.L.B., and National

Institutes of Health Grant T35 DK060444 to K.0.

Correspondence should be addressed Mark L. Baccei at mark.baccei@uc.edu.

https://doi.org/10.1523/JNEUROSCI.0029-20.2020

Copyright $\odot 2020$ the authors

\section{Introduction}

Approximately $8 \%$ of all infants in the United States are admitted to a neonatal intensive care unit after birth (Harrison and Goodman, 2015), where they are subjected to an average of 1315 invasive procedures each day, most of which are considered painful (Simons et al., 2003). Neonatal injury has unique effects on pain processing in both humans and rodents (Walker et al., 2016), potentially because it occurs during a critical period when somatosensory networks are still actively developing (Fitzgerald, 2005). Children previously admitted to the neonatal intensive care unit display long-term increases in pain sensitivity in 
response to prolonged nociceptive stimulation compared with controls (Hermann et al., 2006; Hohmeister et al., 2010). Meanwhile, neonatal surgical injury predisposes adult rodents to increased pain sensitivity (termed "priming") when the same dermatome is reinjured later in life (Walker et al., 2009; Moriarty et al., 2018, 2019), thereby providing a preclinical model system to facilitate exploration of the neurobiological mechanisms by which early-life tissue damage can evoke prolonged changes in nociceptive processing.

The ability of neonatal tissue damage to prime pain sensitivity could involve a persistent sensitization of nociceptive circuits in the spinal superficial dorsal horn (SDH). Indeed, early-life injury heightens glutamatergic signaling in the adult mouse SDH (Li et al., 2015) and increases susceptibility to long-term potentiation (LTP) at sensory synapses onto lamina I spinoparabrachial neurons ( $\mathrm{Li}$ and Baccei, 2016), which represent a key output of the spinal pain network (Todd, 2010). In addition, neonatal injury reduces the efficacy of local inhibitory neurotransmission in the mature SDH (Li et al., 2013, 2015; Li and Baccei, 2019), which includes a weakening of feedforward inhibition (FFI) onto ascending projection neurons following primary afferent input (Li et al., 2015). Elucidating the cellular and molecular mechanisms underlying these deficits in synaptic inhibition is critical to fully understand how neonatal trauma primes developing pain pathways, since disinhibition in the spinal dorsal horn (DH) clearly results in aberrant pain (Melzack and Wall, 1965; Duan et al., 2014; Petitjean et al., 2015).

The DH contains multiple subpopulations of GABAergic interneurons which regulate distinct, yet overlapping, somatosensory modalities (Bourane et al., 2015; Petitjean et al., 2015). For example, interneurons characterized by the expression of dynorphin (DYN) have been implicated in suppressing itch (Kardon et al., 2014; Huang et al., 2018) and mechanical pain (Duan et al., 2014). Furthermore, DYN interneurons are highly prevalent in the DH (Boyle et al., 2017) and synapse directly onto lamina I projection neurons (Hachisuka et al., 2020). As a result, it is possible that persistent deficits in the function of spinal DYN circuits in the aftermath of neonatal tissue damage could contribute to both the reduction in FFI onto adult spinoparabrachial neurons (Li et al., 2015) and the exacerbation of mechanical pain hypersensitivity following subsequent injury, the latter of which is a hallmark feature of neonatal priming (Walker et al., 2009, 2015). Unfortunately, the long-term consequences of early tissue damage for the function of identified interneurons within the spinal nociceptive network have yet to be explored.

Therefore, the present study investigated the effects of neonatal surgical injury on the functional properties of adult mouse $\mathrm{SDH}$ interneurons derived from the DYN lineage, the vast majority of which are GABAergic (Duan et al., 2014). We found that hindpaw incision at postnatal day 3 (P3) compromised multiple components of a putative feedforward inhibitory pathway linking peripheral sensory neurons to mature lamina I projection neurons, by reducing primary afferent drive to the DYN population, lowering intrinsic membrane excitability in DYN neurons, and decreasing the strength of DYN inhibitory synapses onto spinoparabrachial neurons. Collectively, these results identify, for the first time, an inhibitory microcircuit within the adult $\mathrm{SDH}$ that is persistently disrupted by neonatal tissue damage.

\section{Materials and Methods}

Animals

All experiments were performed in accordance with animal welfare guidelines outlined by the Institutional Animal Care and Use Committee at the University of Cincinnati. To identify DYN-lineage interneurons via fluorescence, we crossed Pdyn-IRES-Cre mice (DYN ${ }^{\text {Cre }}$, The Jackson Laboratory; stock \#027958), which express Cre recombinase from the prodynorphin gene promoter, with Ai9 mice (The Jackson Laboratory; stock \#007909), which express a Cre recombinase-dependent tdTomato (tdTOM) from the Rosa26 locus under control of the CAG promoter. The resulting offspring will be referred to as $D Y N^{T O M}$. To record inhibitory DYN input to projection neurons, we used a cross between the $D Y N^{C r e}$ and Ai32 mice (The Jackson Laboratory; stock \#012569), which express a Cre-dependent channelrhodopsin-2 (ChR2)-eYFP fusion protein from the Rosa26 locus. The resulting offspring will be referred to as $D Y N^{C h R}$. In order to genetically label putative DYN presynaptic terminals, we crossed $D Y N^{C r e}$ and Ai34D mice (The Jackson Laboratory; stock \#012570), which express a Cre recombinase-dependent synaptophysintdTOM fusion protein. The resulting offspring will be referred to as $D Y N^{\text {Syp TOM }}$

Adult (P49-P80) female mice were used in this study, as many chronic pain conditions are more prevalent in women (LeResche, 1999). However, a recent study found that neonatal injury reduced sensory drive onto inhibitory interneurons in the adult mouse SDH to a similar degree in males and females ( $\mathrm{Li}$ and Baccei, 2019), suggesting that the long-term changes in inhibitory SDH circuits may not be sex-dependent. Finally, recent efforts to transcriptionally profile DYN interneurons in the spinal DH throughout development found no significant sex differences in gene expression within this population (Serafin et al., 2019).

\section{Hindpaw surgical injury}

Mice were anesthetized with isoflurane $(2 \%-3 \%)$ at P3, and a small incision was made through the skin and underlying muscle of the plantar hindpaw as described previously (Brennan et al., 1996; Li et al., 2013). The skin was immediately closed with 7-0 suture (Ethicon), and the wound fully healed in $\leq 2$ weeks.

\section{Labeling lamina I projection neurons}

To identify projection neurons for immunohistochemical and electrophysiological experiments, mice were given unilateral injections of either cholera toxin subunit B (CTB; 1\%; Sigma Millipore) or FAST DiI oil $(2.5 \mathrm{mg} / \mathrm{ml}$; Thermo Fisher Scientific), respectively. Mice were anesthetized with a mixture of ketamine $(90 \mathrm{mg} / \mathrm{kg})$ and xylazine $(10 \mathrm{mg} / \mathrm{kg})$ in sterile saline $(0.9 \%)$, positioned in a stereotaxic apparatus, and secured with nonrupture ear bars (World Precision Instruments). An incision was made through the scalp to visualize both $\lambda$ and bregma. The following coordinates (in cm relative to bregma) were used to target the parabrachial nucleus, which is innervated by the majority of lamina I projection neurons (Cameron et al., 2015): $-0.47-0.49$ rostrocaudal, -0.12 mediolateral, and $-0.40-0.42$ dorsoventral based on an atlas of the mouse brain (Paxinos and Franklin, 2012). A small hole was drilled in the skull with an OmniDrill35 (World Precision Instruments) and the retrograde tracer CTB $(75 \mathrm{nl}$ at $35 \mathrm{nl} / \mathrm{min}$ ) or DiI ( $150 \mathrm{nl}$ at $35 \mathrm{nl} / \mathrm{min}$ ) was injected into the parabrachial nucleus with a Hamilton syringe (62RN; $2.5 \mu \mathrm{l}$; 33-gauge needle; Hamilton). After the injection, the skin was closed using either Vetbond $(3 \mathrm{M})$ or Gluture (Zoetis), and mice were returned to their home cage for $\sim 1$ week before endpoint electrophysiological or immunohistochemical experiments.

Immunohistochemistry

$D Y N^{T O M}$ or DYN ${ }^{\text {SyPTOM }}$ mice were deeply anesthetized with sodium pentobarbital (Fatal-Plus, Vortech Pharmaceuticals), and 4\% PFA in 0.1 $\mathrm{M} \mathrm{PB}$ was used to transcardially perfuse the mice. The brain and spinal cord lumbar enlargements were removed from the animal and fixed in PFA for $4 \mathrm{~h}$ and then incubated in $30 \%$ sucrose (in $0.1 \mathrm{M}$ phosphate buffer) at $\sim 4^{\circ} \mathrm{C}$ overnight or until the tissue sank. Sections were cut $(40 \mu \mathrm{m})$ on a Leica cryostat and then washed in $0.01 \mathrm{M}$ PBS followed by incubation with blocking buffer $(0.3 \%$ Triton X-100 and 10\% donkey serum; Sigma Millipore). Sections were incubated in a primary antibody against the inhibitory neuron transcription factor Pax2 (1:500 dilution, Thermo Fisher Scientific, \#716000; RRID:AB_2533990) or in antibodies against CTB (1:2000 dilution; List Biological, catalog \#703, RRID:AB_ $10013220)$ and the vesicular GABA transporter VGAT (1:2000 dilution; 
Synaptic Systems, catalog \#131002, RRID:AB_887871) overnight at $4^{\circ} \mathrm{C}$. Sections were then washed in PBS and incubated in a species-specific secondary antibody (AlexaFluor-488; Thermo Fisher Scientific, \#A11008; RRID:AB_143165 and/or AlexaFluor-647; Thermo Fisher Scientific \#A-31 573; RRID:AB_2536183) at a 1:500 dilution for $1 \mathrm{~h}$ at room temperature.

To visualize Pax2 expression in DYN interneurons, the SDH was imaged on a BX63 upright fluorescent microscope (Olympus) at 20$40 \times$; $z$-stack images were taken with a separation of $1 \mu \mathrm{m}$. tdTOMand $\mathrm{tdTOM} / \mathrm{Pax} 2$-expressing cells were counted in 6 sections from each animal and analyzed with CellSens Dimension Desktop software (Olympus).

To evaluate putative DYN presynaptic terminals apposed to projection neurons, images were captured on an inverted confocal microscope (Nikon, A1 LUNA) using a $100 \times$ oil immersion lens. $z$-stack images were sequentially scanned at an interval of $0.5 \mu \mathrm{m}$. The surface area of the cell was estimated by assuming the dendrites were cylindrical ( $\mathrm{A}=2 \pi \mathrm{rh}+2 \pi r^{2}$; where $\mathrm{h}=$ height and $r=$ radius), and the soma to have an ellipsoid shape with $A=4 \pi\left(\frac{(a b)^{1.6}+(a c)^{1.6}+(b c)^{1.6}}{3}\right)^{1 / 1.6}$ as described previously (Todd et al., 2002). After a $z$-series of images captured the majority of the neuron and its dendritic arbor, boutons containing tdTOM (corresponding to the terminals of DYN-lineage neurons) and/or VGAT (corresponding to inhibitory terminals) were counted in each image if the terminals were directly apposed to the dendrites or soma of the CTB-labeled projection neuron, similar to the approach we previously used to quantify putative inhibitory synaptic contacts onto lamina I spinoparabrachial neurons (Li et al., 2015). The number of $\mathrm{tdTOM}^{+}$and $\mathrm{tdTOM}^{+} /$ $\mathrm{VGAT}^{+}$boutons are presented as the number of contacts per $1000 \mu \mathrm{m}^{2}$ area of the projection neuron. Presumed contacts were only quantified in areas where the projection neuron membrane could be clearly outlined. Images were analyzed using Nikon Elements and ImageJ/Fiji software, and both Inkscape and GraphPad Prism (version 8; GraphPad Software) were used to create the figures.

\section{Spinal cord slice preparation}

All solutions that contacted live tissue were bubbled with carbogen (95\% oxygen, $5 \%$ carbon dioxide). Mice were deeply anesthetized using sodium pentobarbital (Fatal-Plus; Vortech Pharmaceuticals) and transcardially perfused with ice-cold sucrose-substituted artificial cerebrospinal fluid (dissection solution; containing the following, in mM: 250 sucrose, 2.5 $\mathrm{KCl}, 25 \mathrm{NaHCO}_{3}, 1.0 \mathrm{NaH}_{2} \mathrm{PO}_{4}, 6 \mathrm{MgCl}_{2}, 0.5 \mathrm{CaCl}_{2}$, and 25 glucose). The vertebral column was removed, submerged in ice-cold dissection solution, and the spinal cord isolated. The dura and pia mater were stripped from the spinal cord. Dorsal roots were removed from the spinal cord, with the exception of experiments involving the analysis of sensory innervation to DYN interneurons in which the $\mathrm{L} 3$ and $\mathrm{L} 4$ roots were left intact. The spinal cord was submerged in warm $3 \%$ low-melting point agarose (Invitrogen) dissolved in dissection solution and chilled to solidify. Parasagittal slices $(250-400 \mu \mathrm{m})$ were cut in ice-cold dissection solution on a vibrating microtome (7000smz-2; Campden Instruments). Then slices recovered at room temperature for 15-20 $\mathrm{min}$ in a solution containing the following (in mM): $92 \mathrm{NMDG}, 2.5 \mathrm{KCl}, 1.2 \mathrm{NaH}_{2} \mathrm{PO}_{4}, 30 \mathrm{NaHCO}_{3}$, 20 HEPES, 25 glucose, $5 \mathrm{Na}$ ascorbate, 2 thiourea, $3 \mathrm{Na}$ pyruvate, 10 $\mathrm{MgSO}_{4}$, and $0.5 \mathrm{CaCl}_{2}$ (Ting et al., 2014). After recovering, slices were transferred to artificial cerebrospinal fluid (aCSF; composed of the following, in mM: $125 \mathrm{NaCl}, 2.5 \mathrm{KCl}, 25 \mathrm{NaHCO}_{3}, 1.0 \mathrm{NaH}_{2} \mathrm{PO}_{4}, 1.0 \mathrm{MgCl}_{2}, 2.0$ $\mathrm{CaCl}_{2}$, and 25 glucose) to incubate at room temperature until recording.

Patch-clamp recordings

Slices were transferred to a submersion chamber (RC-22; Warner Instruments) after incubation, then secured with an anchor, and placed on the stage of an upright microscope (BX51WI; Olympus). Slices were constantly perfused with oxygenated aCSF at room temperature during recordings. DYN interneurons in laminae I-II were identified via tdTOM fluorescence and projection neurons via DiI fluorescence, and both were visualized for patch-clamp recording via infrared LED illumination. Thin-walled single-filament borosilicate glass ( $1.5 \mathrm{~mm}$ diameter, World Precision Instruments) was used for patch pipettes, which were created in a microelectrode puller (P-97; Sutter Instruments). The resistance of patch pipettes was 3-7 M $\Omega$, with a minimum seal resistance of 1 $\mathrm{G} \Omega$ obtained before entering whole-cell mode. A Multiclamp 700B amplifier (Molecular Devices) and Digidata 1440A digitizer were used with pClamp 10.4 software (Molecular Devices) to acquire recordings. Liquid junction potential was accounted for $(-14 \mathrm{mV})$, which was calculated using JPCalc software (P. Barry, University of New South Wales, Sydney, Australia; modified for Molecular Devices). A $4 \mathrm{kHz}$ low-pass Bessel filter and $10 \mathrm{kHz}$ sampling rate were used when acquiring recordings.

To characterize the pattern of primary afferent input to DYN interneurons, as well as their intrinsic membrane excitability, a $\mathrm{K}^{+}$gluconate-based intracellular solution was used (composed of the following, in $\mathrm{mM}$ : $130 \mathrm{~K}$-gluconate, $10 \mathrm{KCl}, 10 \mathrm{HEPES}, 10 \mathrm{Na}$-phosphocreatine, $0.3 \mathrm{Na}_{2}$-GTP, and $2 \mathrm{MgATP}, \mathrm{pH}$ 7.2, 295-305 mOsm). All measurements of the intrinsic membrane excitability of DYN interneurons were performed in current clamp. Intrinsic action potential (AP) discharge was evoked from the resting membrane potential via intracellular current injections (-20-130 pA in $10 \mathrm{pA}$ steps, $800 \mathrm{~ms}$ duration). Instantaneous firing frequency was calculated (in $\mathrm{Hz}$ ) as 1 /mean interspike interval. Rheobase was measured as the minimum current step (delivered in $2.5 \mathrm{pA}$ increments at 50 ms duration) that evoked an AP.

For all recordings involving primary afferent input, the dorsal root (L3 or L4) was stimulated via a suction electrode using a constant current stimulator (Master-8; A.M.P.I.) with a stimulus isolation unit (ISOFlex; A.M.P.I.). DYN neurons were held at $-70 \mathrm{mV}$ in voltage clamp, or recorded from their resting membrane potential in current clamp, and the $\mathrm{L} 3$ or $\mathrm{L} 4$ dorsal root $(7-10 \mathrm{~mm}$ in length) was stimulated via a suction electrode at 10 incrementally increasing intensities $(0,10,20,30,50$, $100,150,300,500 \mu \mathrm{A}$, and $1 \mathrm{~mA} ; 100 \mu$ s duration; $0.1 \mathrm{~Hz}$ ). The threshold to evoke an excitatory postsynaptic current (EPSC) was defined as the current intensity which evoked a measurable EPSC in $\geq 50 \%$ of the trials. The stimulus threshold and onset latency of an evoked EPSC were jointly used to classify the observed synaptic response as mediated by $\mathrm{A} \beta$ fibers, $\mathrm{A} \delta$ fibers, low-threshold $\mathrm{C}$ fibers, or high-threshold $\mathrm{C}$ fibers, guided by information subsequently gained from compound APs recorded from the dorsal roots, as described previously (Li et al., 2015). EPSCs mediated by A $\beta$ fibers were classified as monosynaptic based on their ability to follow repetitive stimulation $(20 \mathrm{~Hz})$ with a constant latency and absence of failures, whereas $\mathrm{A} \delta$ fiber- and $\mathrm{C}$ fiber-mediated EPSCs were considered monosynaptic if no failures were observed during 2 and $1 \mathrm{~Hz}$ stimulation, respectively (Torsney and MacDermott, 2006). To record the compound APs, the dorsal root was severed near the entry zone at the end of the experiment and placed into two suction electrodes for stimulation and recording, and compound APs were generated via incremental stimulation $(0-1 \mathrm{~mA}, 100 \mu$ s duration) of the dorsal root fibers. Importantly, we have previously shown that neonatal incision fails to alter the recruitment thresholds or conduction velocities of adult dorsal root fibers (Li et al., 2015).

The occurrence of FFI in DYN neurons was first determined in voltage clamp (from a holding potential of $-60 \mathrm{mV}$ ) by the appearance of an outward component of the overall postsynaptic current evoked by primary afferent stimulation. Next, the dorsal root was stimulated at increasing intensities ( $10 \mu \mathrm{A}$ to $1 \mathrm{~mA}$ ) in the current-clamp configuration to determine whether AP discharge occurred in the sampled neuron. AP firing was quantified as the number of APs discharged in a $1 \mathrm{~s}$ period following dorsal root stimulation. In separate experiments, inhibitory postsynaptic currents (IPSCs) were evoked in DYN neurons by dorsal root stimulation from a holding potential of $0 \mathrm{mV}$ using an intracellular solution containing the following (in $\mathrm{mM}$ ): 130 Cs-gluconate, $10 \mathrm{CsCl}, 10$ HEPES, 11 EGTA, $1 \mathrm{CaCl}_{2}$, and $2 \mathrm{MgATP}$, pH 7.2 (295-305 mOsm).

To measure inhibitory DYN input to projection neurons, spinal cord slices were prepared from $D Y N^{C h R}$ mice as described above and patchclamp recordings obtained from DiI-labeled lamina I projection neurons using an intracellular Cs-gluconate solution as described above. First, projection neurons were voltage clamped at $-70 \mathrm{mV}$, and blue light was applied $(470 \mathrm{~nm}, 10 \mathrm{~ms}$; CoolLED) to the portion of the spinal cord preparation that fell within the visual field using the $40 \times$ objective on the microscope, to confirm a lack of ChR2 expression in the recorded projection neuron. Next, antagonists for AMPA (NBQX, $10 \mu \mathrm{M}$ ) and 
NMDA receptors (AP5, $20 \mu \mathrm{M})$ were administered via the bath to isolate IPSCs. Projection neurons were then voltage-clamped at $0 \mathrm{mV}$, and IPSCs were evoked by stimulating DYN neurons via blue light $(470 \mathrm{~nm}$, $10 \mathrm{~ms}$ ). To investigate the properties of presynaptic neurotransmitter release from DYN interneurons, the coefficient of variation was measured by delivering 30 light pulses at $0.1 \mathrm{~Hz}$ and calculating the $\mathrm{SD} /$ mean amplitude of the DYN-evoked IPSCs. The paired-pulse ratio (PPR) was calculated as the mean amplitude of IPSC2/mean amplitude of IPSC1 after delivering two identical pulses of light at an interval of $75 \mathrm{~ms}$ (for 5 trials).

\section{Clustering analysis}

Unbiased clustering analysis based on intrinsic membrane properties was conducted on a dataset composed of recordings obtained from laminae I-II neurons from adult Gad67-EGFP mice (The Jackson Laboratory, stock \#003718), which were sampled as part of a prior study (Li and Baccei, 2014), pooled with the current recordings from adult $D Y N^{T O M}$ mice. In both studies, cells were recorded from mice which had either incision or isoflurane exposure only (as a control) at P3. The electrophysiological features used for this analysis were membrane capacitance, resting membrane potential, membrane resistance, and rheobase. All features were centered and scaled (i.e., $z$-scored) before analysis. Each cell was treated as one observation with each of the above specified features as a variable.

Principal component analysis was conducted in $\mathrm{R}$ using base function $\operatorname{prcomp}()$ and package factoextra (Kassambara and Mundt, 2019). $k$-means clustering was conducted using R packages factoextra and cluster (Maechler et al., 2018). Using the Silhouette method (Rousseeuw, 1987) with Euclidean distance metric, the optimal number of clusters was determined to be 4 . Therefore, $k$-means clustering using Euclidean distance was conducted using the $k$-means function with centers $=4$ and nstart $=25$.

\section{Experimental design and statistical analysis}

The immunohistochemical data shown in Figure $1 B$ were taken from 6 adult mice ( 3 naive and 3 with neonatal incision) with 6 sections used from each mouse, and the percent of Pax2/DYN-tdTOM colocalization was analyzed with a repeated-measures two-way ANOVA with the factors neonatal injury $\times$ side of the spinal cord respective to the injury, followed by Sidak's multiple comparisons test to determine individual group differences.

The repetitive firing frequency dataset (see Fig. 2B) includes 13 neurons taken from 2 naive adult mice and 12 neurons taken from 2 neonatally incised adult mice, and was analyzed with a repeated-measures two-way ANOVA (factors: neonatal injury $\times$ stimulus intensity as the repeated measure) followed by Sidak's multiple comparisons test. The resting membrane potential measurements (see Fig. 2C) were taken from 22 neurons in 3 naive mice and 26 neurons in 3 neonatally injured mice; these data were analyzed with a nested unpaired $t$ test. The experiments illustrated in Figure 2D involved measuring membrane resistance from 21 neurons from 3 naive mice and 24 neurons from 3 mice with P3 incision, and data were analyzed with a nested unpaired $t$ test (after transforming the data). Rheobase (see Fig. 2E) was measured in 17 neurons taken from 2 mice in each group, and the data were analyzed with a nested unpaired $t$ test (after transforming the data).

Experiments designed to characterize primary afferent-evoked glutamatergic drive to DYN interneurons involved 37 neurons sampled from 9 naive mice and 26 neurons recorded from 8 neonatally incised mice, which were used to classify the pattern of primary afferent input (see Fig. 3B). In Figure 3C, 24 cells were used from 4 naive mice and 18 cells were taken from 3 neonatally incised mice. Because of missing values at a single stimulus intensity $(1000 \mu \mathrm{A})$ in 3 cells from the naive group, these data were analyzed with a mixed-effects model (factors: injury $x$ stimulus intensity as the repeated measure) followed by Sidak's post hoc test to determine individual group differences. The amplitude of monosynaptic primary afferent input was measured in 33 neurons taken from 9 naive mice and 27 neurons taken from 8 mice with P3 incision and analyzed with the Mann-Whitney test.

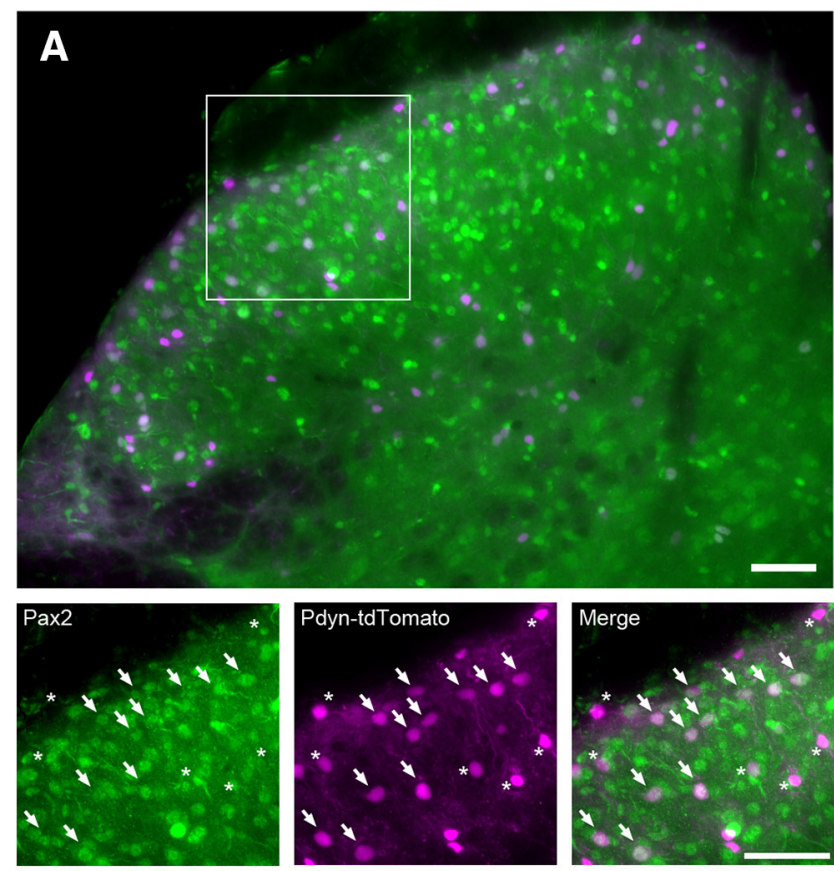

\section{B}

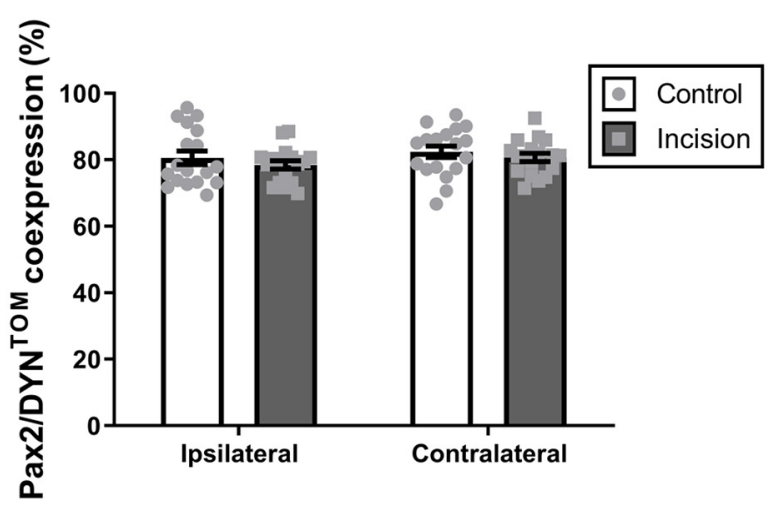

Figure 1. DYN ${ }^{\text {Cre }}$ predominantly marks inhibitory neurons in the adult DH regardless of neonatal incision. $\boldsymbol{A}$, Example of the distribution of the inhibitory neuron marker Pax2 (green) and DYN-tdTOM (magenta) in the adult mouse DH. Scale bar, $50 \mu \mathrm{m}$. $\boldsymbol{B}$, Colocalization analysis revealed no significant effect of neonatal incision on the percentage of spinal DYN-tdTOM interneurons that exhibit an inhibitory phenotype ( $n=18$ sections; $F_{(1,34)}=0.91, p=0.35$ for injury, repeated-measures two-way ANOVA).

To evaluate the appearance of FFI onto DYN interneurons near their resting membrane potential (see Fig. $4 C$ ), 44 neurons from 7 naive mice and 33 neurons from 6 neonatally incised mice were used, and the Fisher's exact test was used to detect a difference in the distributions. To evaluate the relationship between FFI and AP firing in DYN interneurons (see Fig. 4D), 26 neurons from 3 naive mice and 19 neurons from 3 neonatally incised mice were used, and data were analyzed with a Fisher's exact test. Finally, as a more direct measure of FFI, primary afferent-evoked IPSCs were recorded at a holding potential of $0 \mathrm{mV}$ in 18 neurons from 4 naive adult mice and 14 neurons from 3 adult mice with P3 incision.

In the analysis of dorsal root-evoked firing in adult DYN interneurons (see Fig. $5 B$ ), there were 24 cells sampled from 4 naive mice and 18 cells recorded from 3 neonatally incised mice. Because of missing values at a single stimulus intensity $(1000 \mu \mathrm{A})$ in 3 cells from the naive group, data were analyzed with a mixed-effects model (factors: P3 incision $\times$ stimulus intensity as the repeated measure) followed by Sidak's multiple comparisons test to determine differences between individual groups. 
A
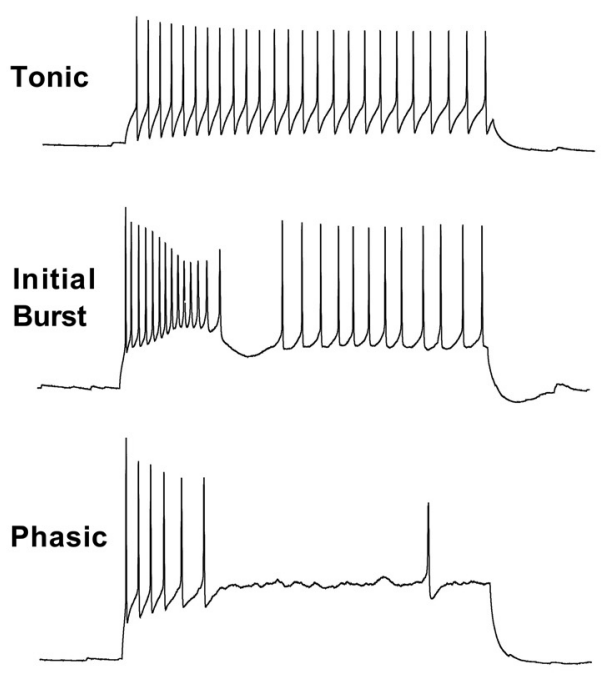

B

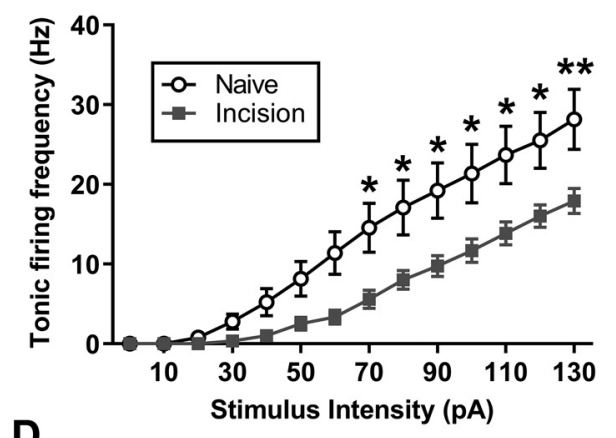

D

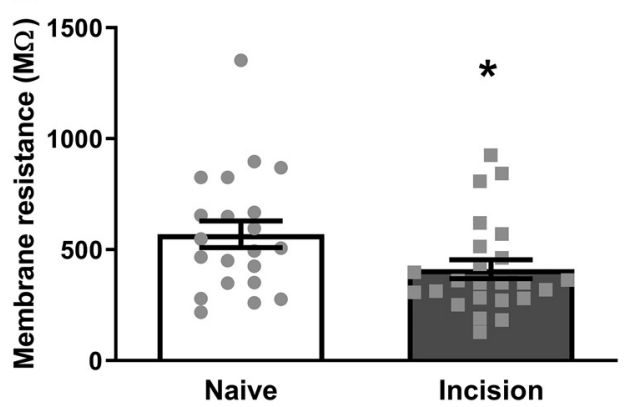

Incision
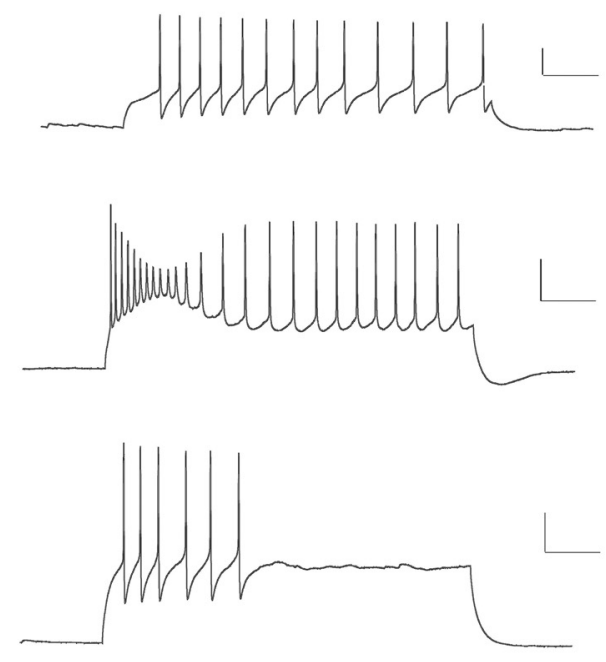

C

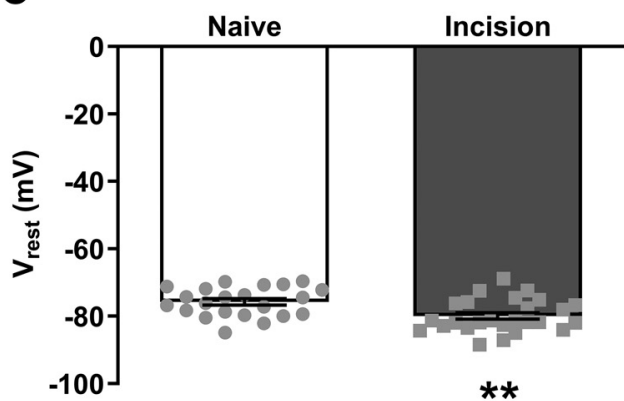

E

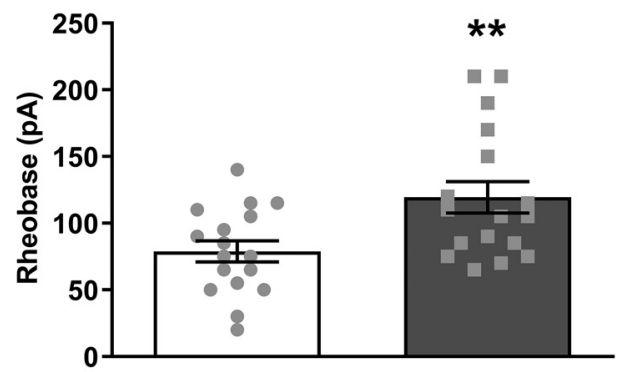

Naive

Incision

Figure 2. Neonatal incision reduces the intrinsic excitability of adult DYN interneurons. $A$, Representative traces of intrinsic firing in DYN interneurons. Within the tonic firing group (top), firing is more frequent in naive (left) versus neonatally incised mice (right). Calibration, $A-E: 20 \mathrm{mV}, 100 \mathrm{~ms}$. B. Plot of repetitive firing frequency as a function of stimulus intensity revealing a lower intrinsic firing frequency in DYN interneurons from adult mice with prior neonatal incision (naive: $n=13$ neurons; incision: $n=12 ; F_{(13,299)}=5.296, p<0.0001$ for injury $\times$ stimulus intensity, repeated-measures two-way ANOVA). ${ }^{*} p<0.05$; ${ }^{* *} p<0.01$; Sidak's multiple comparisons test. $C$, DYN interneurons sampled from adult mice with prior neonatal incision display a more hyperpolarized resting potential compared with naive littermate controls (naive: $n=22$ neurons; incision: $n=26 ; t=3.15 ; * * p=0.0029$; nested unpaired $t$ test). D, Membrane resistance in mature DYN interneurons is significantly reduced by prior neonatal incision (naive: $n=21$; incision: $n=24 ; t=2.32$; ${ }^{*} p=0.025$; nested unpaired $t$ test). $\boldsymbol{E}$, Neonatal incision also increases the mean rheobase in adult DYN interneurons ( $n=17$ neurons in each group; $t=2.88 ;{ }^{* *} p=0.007$; nested unpaired $t$ test).

Experiments evaluating the DYN synaptic input to adult projection neurons (see Fig. 6) consisted of 20 projection neurons in each group (from 10 naive mice and 7 mice with P3 incision). IPSC amplitudes (see Fig. $6 B$ ) were analyzed with a nested unpaired $t$ test (after transforming the data), whereas the coefficient of variation data (see Fig. $6 C$ ) and PPRs (see Fig. 6D) were analyzed with nested unpaired $t$ tests.

To anatomically examine the number of putative DYN contacts onto projection neurons (see Fig. 7), we analyzed 9 adult projection neurons from 3 naive mice and 12 neurons from 4 neonatally incised mice. The data were separated into inhibitory and presumed excitatory DYN boutons in apposition to either the dendrites or soma of the projection neurons, and analyzed via nested unpaired $t$ tests in the absence (see Fig. $7 B$, right, $C$ ) or presence (see Fig. $7 B$, left) of data transformation into a normal distribution.

The clustering analysis shown in Figure $8 A, B$, used a total of 85 neurons distributed across the following experimental groups: Gad-GFP naïve, $n=25$ cells from 3 female Gad67-EGFP mice; Gad-GFP incision, $n=26$ cells from 3 female Gad67-EGFP mice; DYN naïve, $n=17$ cells from $3 D Y N^{T O M}$ mice; and DYN incision, $n=17$ cells from $3 D Y N^{T O M}$ mice. Comparison of each electrophysiological feature between the different clusters was conducted using the Kruskal-Wallis test. Meanwhile, to statistically compare the effects of neonatal incision on primary 
A
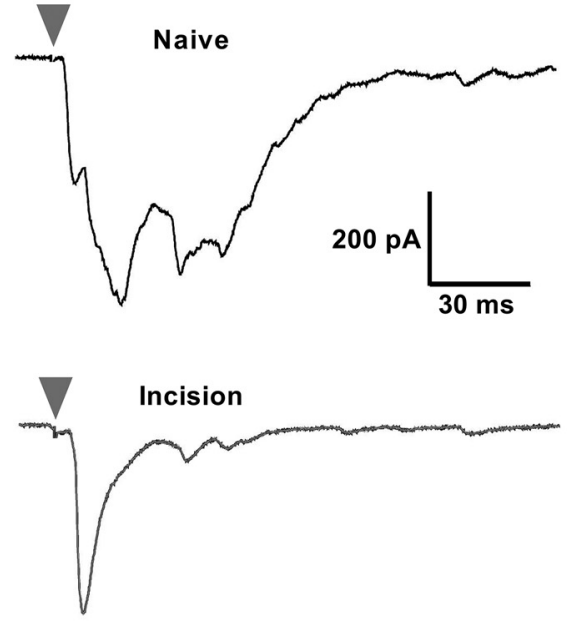

B

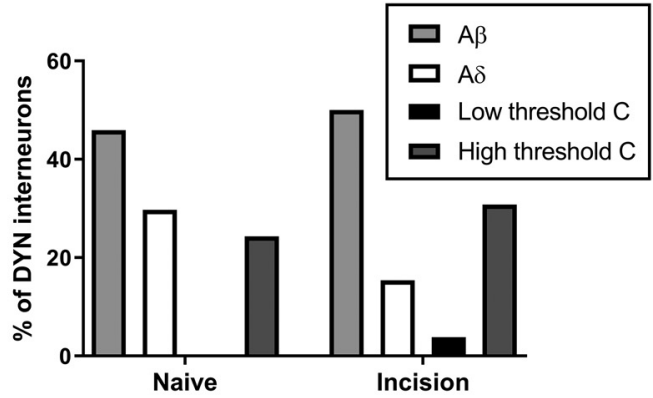

C

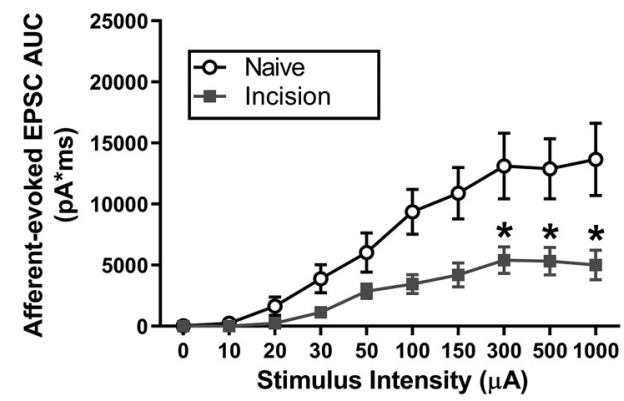

Figure 3. Neonatal injury weakens primary afferent-evoked glutamatergic drive to DYN interneurons in adulthood. $\boldsymbol{A}$, Representative traces of dorsal root-evoked EPSCS in DYN-tdTOM neurons from naive mice (top; gray triangle represents stimulus onset in all panels), which are reduced by early-life tissue damage (bottom). $\boldsymbol{B}$, There was no obvious difference in the types of monosynaptic primary afferent input received by adult DYN interneurons in naive versus neonatally incised littermates (naive: $n=37$ neurons; P3 incision: $n=26$ neurons). C, Total afferent-evoked glutamatergic drive to adult DYN interneurons was significantly diminished by neonatal injury (naive: $n=24$; incision: $n=18$; $F_{(9,355)}=25.33, p<0.0001$ for injury $\times$ stimulus intensity, mixed-effects model), with a lower EPSC area under the curve (AUC) in mice that underwent P3 incision. ${ }^{*} p<0.05$; Sidak's multiple comparisons test.

afferent-evoked glutamatergic input (see Fig. $8 C$ ) and AP discharge (see Fig. $8 D$ ) in DYN neurons to those effects seen across the wider GABAergic population, we included previously collected data from 15 GABAergic (i.e., Gad67-GFP) neurons from naive mice and 12 GABAergic neurons from neonatally incised mice (Li and Baccei, 2019) and compared these results with the present data obtained from DYNtdTOM neurons (see Figs. 3C, 5B) using mixed-effects models.

All data, including those illustrated in the figures, are expressed as mean \pm SEM unless otherwise stated.

\section{Results}

\section{Early-life injury lowers the intrinsic excitability of spinal DYN interneurons in adulthood}

While neonatal tissue damage reduces the intrinsic excitability of GABAergic interneurons in the adult mouse $\mathrm{SDH}$ ( $\mathrm{Li}$ and Baccei, 2014), it is unclear which subpopulations of inhibitory neurons are persistently affected by surgical injury during early life. Accordingly, we evaluated the intrinsic membrane properties of mature DYN interneurons after P3 incision. Previous data demonstrate that DYN-lineage neurons in the $\mathrm{DH}$ (identified via Cre recombinase-mediated tdTOM expression in $D Y N^{T O M}$ mice) are predominantly inhibitory interneurons, as defined by extensive coexpression of GAD67 (Duan et al., 2014) or Pax2 (C.L.B., L. Styczynski, E.K.S., M.L.B., unpublished observations; Serafin et al., 2019), the latter of which is present in all inhibitory neurons in the DH (Larsson, 2017). Furthermore, a significant depletion of Tlx3, which specifies a glutamatergic fate in the $\mathrm{DH}$ (Cheng et al., 2004), was observed in spinal DYN-lineage cells (Serafin et al., 2019).

Nonetheless, since a phenotypic shift in the pattern of DYN expression within the developing $\mathrm{SDH}$ after neonatal injury could complicate the interpretation of the below experiments, we first investigated the effect of P3 hindpaw incision on the extent of tdTOM/Pax2 colocalization in the adult mouse SDH (Fig. $1 A)$. Consistent with our previous findings, the majority $(\sim 80 \%)$ of DYN-tdTOM neurons in the mature SDH expressed Pax2 (Fig. 1B). More importantly, we found a similar expression of Pax2 within DYN-lineage neurons in neonatally injured mice compared with naive littermate controls $(n=18$ sections; $F_{(1,34)}=0.91, p=0.35$ for the main effect of injury, repeatedmeasures two-way ANOVA; Fig. 1B). Collectively, these results validate the use of $D Y N^{C r e}$ mice to visualize a subset of GABAergic SDH interneurons under normal or pathologic conditions.

We next analyzed the firing pattern of adult DYN interneurons (Fig. 2A) and found that the majority were tonic firing $(\sim 75 \%)$ with no significant differences in the distribution of firing patterns between naive and neonatally incised mice (data not shown). However, tonic-firing DYN interneurons displayed a lower firing frequency after $\mathrm{P} 3$ hindpaw incision compared with naive littermate controls (naive: $n=13$ neurons; incision: $n=12$; $F_{(13,299)}=5.296, p<0.0001$ for injury $\times$ stimulus intensity, repeated-measures two-way ANOVA; Fig. 2B). Additionally, DYN interneurons from adult mice with prior neonatal incision displayed significantly more hyperpolarized resting membrane potentials (naive: $-75.8 \pm 0.9 \mathrm{mV}, n=22$; incision: $-79.9 \pm$ $0.9 \mathrm{mV}, n=26$; $t=3.15$; $p=0.0029$; nested unpaired $t$ test; Fig. 2C). Finally, neonatal incision lowered the membrane resistance of adult DYN interneurons (naive: $n=21$; incision: $n=24$; $t=2.32 ; p=0.025$; nested unpaired $t$ test; Fig. $2 D$ ), which may have resulted in the long-term increase in rheobase observed after early surgical injury $(n=17$ neurons in each group; $t=2.88 ; p=0.007$; nested unpaired $t$ test; Fig. $2 E$ ). Collectively, these results suggest that neonatal injury persistently reduces the intrinsic excitability of a sizeable population of adult SDH interneurons that are mostly inhibitory in nature. 
A
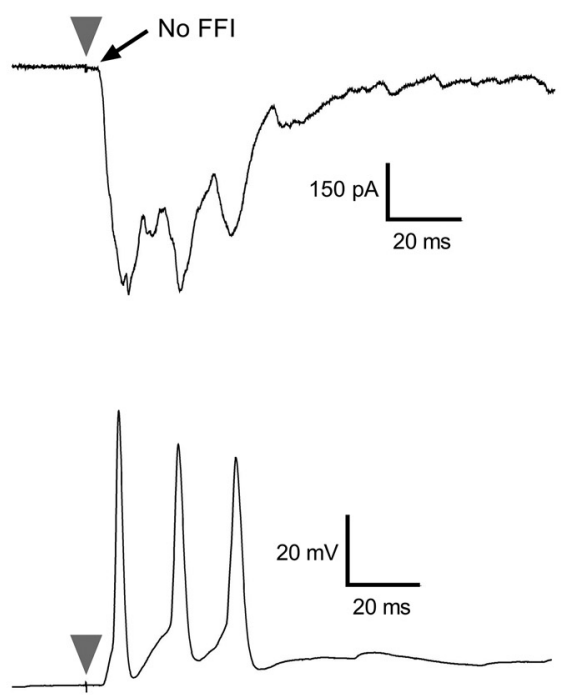

C

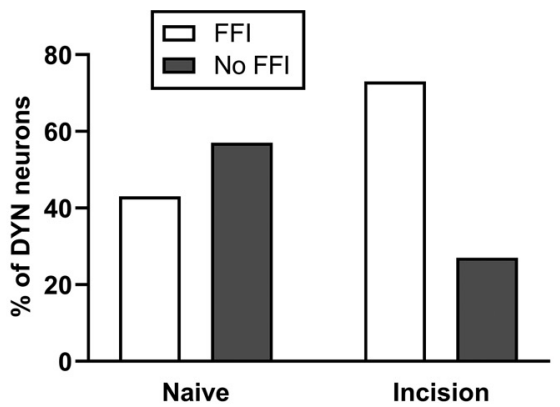

B Incision
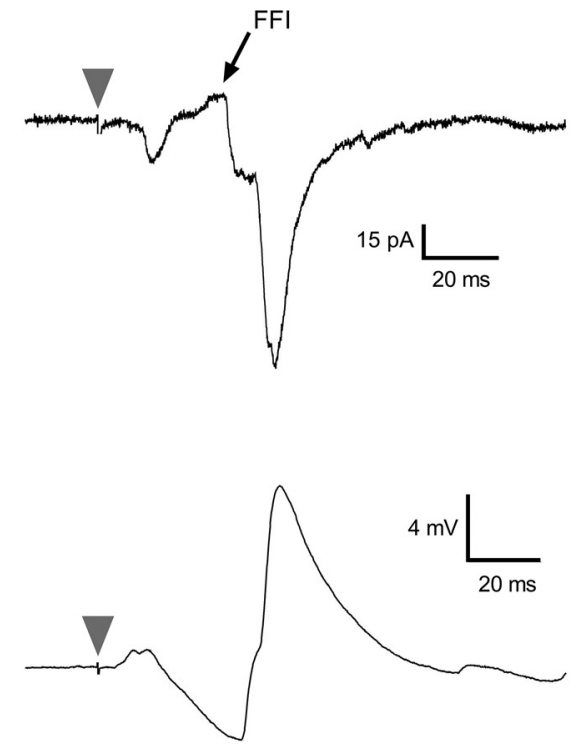

D

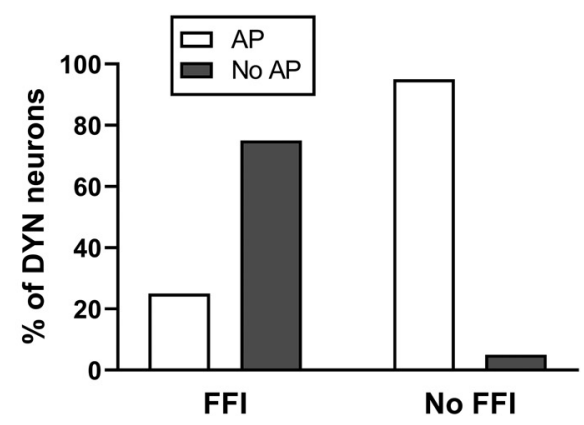

Figure 4. Altered synaptic integration within mature spinal DYN interneurons after early surgical injury. $\boldsymbol{A}$, Representative synaptic currents (top; from a holding potential of $-60 \mathrm{mV}$ ) and corresponding AP discharge (bottom) evoked by dorsal root stimulation (100 $\mu \mathrm{A}, 100 \mu \mathrm{s})$ in an adult DYN-tdTOM DH neuron from a naive mouse, which lacks evidence of FFI (No FFl; black arrow). Gray triangle represents stimulus onset in all panels. $\boldsymbol{B}$, Representative voltage-clamp (top; from a holding potential of $-60 \mathrm{mV}$ ) and currentclamp (bottom) traces illustrating evidence of FFI (top, black arrow) and a corresponding absence of AP firing (bottom) following dorsal root stimulation (300 $\mu \mathrm{A}, 100 \mu \mathrm{s}$ ) in a mature DYN interneuron after P3 incision. Bottom, FFI appears as a hyperpolarizing inhibitory postsynaptic potential (IPSP) that precedes a larger depolarizing excitatory postsynaptic potential (EPSP). C, The appearance of FFI in adult DYN interneurons is significantly more frequent after neonatal incision (right; naive: $n=44 \mathrm{neurons;}$ incision: $n=33$; $p=0.012$; Fisher's exact test). $\boldsymbol{D}$, Primary afferent-evoked AP discharge was significantly less common in those DYN interneurons with measurable FFI (left) compared with DYN interneurons without signs of FFI (right; $n=21-24$ neurons; Fisher's exact test, $p<0.0001$ ).

Long-term reduction in the ability of sensory afferents to drive activity in spinal DYN interneurons after neonatal incision

To determine whether the functional denervation of GABAergic interneurons in the adult mouse SDH following neonatal incision ( $\mathrm{Li}$ and Baccei, 2019) includes a reduction in sensory drive to the DYN neuronal population, we examined the effects of P3 incision on primary afferent-evoked synaptic transmission and AP firing in mature DYN-tdTOM neurons. First, EPSCs were evoked in DYN-tdTOM neurons via electrical stimulation of the attached dorsal root in naive (Fig. $3 A$, top) and neonatally incised mice (Fig. $3 A$, bottom). The types of monosynaptic primary afferent input received by adult DYN interneurons did not significantly change after early-life injury (naive: $n=37$ neurons; P3 incision: $n=26$ neurons; Fig. $3 B$ ). Notably, $\mathrm{A} \beta$ fiber inputs were the most prevalent in both experimental groups, which is in general agreement with previous observations that a considerable proportion $(\sim 27 \%)$ of DYN interneurons in laminae $\mathrm{I}_{-} \mathrm{II}_{\text {outer }}$ of the mature mouse $\mathrm{SDH}$ receive direct synaptic input from $\mathrm{A} \beta$ fibers (Duan et al., 2014). Furthermore, the mean amplitude of the monosynaptic EPSCs was unaffected by neonatal incision (naive: $210.2 \pm 28.7 \mathrm{pA}, n=33$; incision: $242.1 \pm 62.6 \mathrm{pA}, n=27$; $U=401, p=0.51$, Mann-Whitney test; data not shown). However, the overall afferent-evoked (i.e., both monosynaptic and polysynaptic) glutamatergic input to DYN interneurons is markedly reduced in adulthood when preceded by neonatal incision (naive: $n=24$; incision: $n=18 ; F_{(9,355)}=25.33, p<0.0001$ for injury $\times$ stimulus intensity, mixed-effects model; Fig. $3 C$ ). Collectively, these data suggest that the attenuated sensory drive to mature DYN interneurons might predominantly reflect weaker recruitment of polysynaptic excitatory pathways after early injury.

Whether an individual DYN neuron will be activated by sensory input is governed by how the above excitatory synaptic conductances are integrated with inhibitory signals at membrane potentials near the AP threshold. Indeed, inhibitory interneurons within the CNS can form reciprocal connections or silence other inhibitory subpopulations (Pfeffer et al., 2013; Xu et al., 2013; 
A

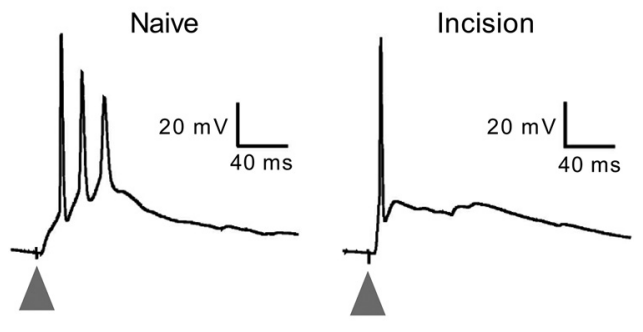

B

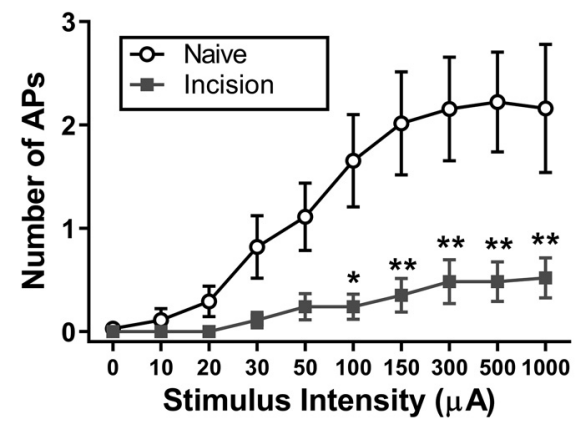

Figure 5. Neonatal tissue damage persistently reduces sensory-evoked output of spinal DYN interneurons. $\boldsymbol{A}$, Representative current-clamp traces demonstrating dorsal root-evoked AP firing in adult DYN neurons from naive mice (left) and mice with prior neonatal incision (right). $\boldsymbol{B}$, Plot of AP discharge versus intensity of dorsal root stimulation revealed reduced afferent-evoked firing in DYN-tdTOM neurons following surgical injury at P3 (naive: $n=24$; incision: $n=18 ; F_{(9,357)}=5.47 ; p<0.0001$ for injury $\times$ stimulus intensity, mixed-effects model). ${ }^{*} p<0.05 ;{ }^{* *} p<0.01$; Sidak's multiple comparisons test.
Roux and Buzsáki, 2015), which can ultimately lead to disinhibition in a neural network. As a result, we next investigated the degree to which P3 incision modulated the integration of primary-afferent evoked synaptic inputs within mature DYNtdTOM neurons near their resting membrane potential, as well as the relationship between the appearance of afferent-evoked FFI in this neuronal population and their ability to discharge APs. The presence of FFI was determined in the voltage-clamp mode by the appearance of an outward component of the overall postsynaptic current evoked by dorsal root stimulation from a holding potential of $-60 \mathrm{mV}$ (Fig. $4 A, B$, top). Importantly, FFI was more likely to be observed in DYN-tdTOM neurons from adult mice with prior neonatal incision (P3 incision: 24 of 33 neurons) compared with naive littermate controls (naive: 19 of 44 neurons; $p=0.012$, Fisher's exact test; Fig. $4 C$ ). Furthermore, current-clamp recordings from the same neurons showed that DYN interneurons exhibiting FFI were significantly less likely to fire APs in response to primary afferent input across the range of stimulus intensities examined (AP discharge with FFI: 6 of 24 neurons; AP discharge without FFI: 20 of 21 neurons; $p<0.0001$, Fisher's exact test; Fig. $4 D$ ).

However, it should be noted that these experiments do not reflect a direct measure of feedforward synaptic inhibition onto DYN neurons. Indeed, the increased appearance of FFI (Fig. 4C) might solely reflect the significant reduction in primary afferentevoked glutamatergic drive to this population after early injury (Fig. 3C), which would unmask many IPSCs within the overall synaptic response. To address this issue further, in separate experiments, we isolated primary afferent-evoked IPSCs using a holding potential of $0 \mathrm{mV}$ (i.e., at the reversal potential for EPSCs) in adult DYN neurons from naive or P3-incised mice as a more direct measure of FFI. While we observed a trend toward an increased prevalence of FFI after neonatal incision, as 11 of 14 DYN neurons from P3incised mice exhibited primary afferentevoked IPSCs compared with only 8 of 18 DYN neurons from naive mice, this failed to reach statistical significance ( $p=0.075$; Fisher's exact test). Based on the available evidence, we conclude that an increase in the appearance of FFI in mature DYN neurons after neonatal incision, which is likely secondary to a reduction in glutamatergic input to this population, is associated with a reduced likelihood of AP discharge.

Given the above disruption in the balance of excitatory versus inhibitory synaptic signaling onto adult DYN neurons after early-life incision (Figs. 3, 4), combined with the persistent reduction in their intrinsic membrane excitability (Fig. 2), we hypothesized that neonatal injury would decrease primary afferentdriven AP firing in this population. Indeed, P3 incision significantly reduced afferent-evoked AP discharge in adult DYN interneurons (naive: $n=24$; incision: $n=18 ; F_{(9,357)}=5.47, p<0.0001$ for injury $\times$ stimulus intensity, mixedeffects model; Fig. 5). These results
Figure 6. Inhibition of spinal projection neurons by DYN interneurons is persistently compromised by neonatal incision. $\boldsymbol{A}$, Examples of IPSCs recorded in a lamina I spinoparabrachial neuron from naive (top) and neonatally incised (bottom) mice in response to the optogenetic stimulation of spinal DYN neurons. $\boldsymbol{B}$, The mean amplitude of DYN-evoked IPSCs in adult projection neurons is significantly decreased by neonatal incision $\left(n=20\right.$ projection neurons in each group; $t=2.32$; ${ }^{*} p=0.035$; nested unpaired $t$ test). C, The IPSCs also displayed a higher mean coefficient of variation after neonatal incision $(n=20$ per group $t=2.77 ;{ }^{*} p=0.014$; nested unpaired $t$ test), suggesting a lower probability of GABA release and/or reduced number of transmitter release sites at DYN inhibitory synapses onto mature projection neurons. $\boldsymbol{D}$, An increase in the PPR of DYN-evoked IPSCS in projection neurons was also observed following P3 hindpaw incision, suggesting a lower probability of GABA release from the presynaptic terminals of inhibitory DYN interneurons in the aftermath of neonatal tissue damage $(n=20$ neurons per group; $t=2.34 ;{ }^{*} p=0.034$; nested unpaired $t$ test).
B

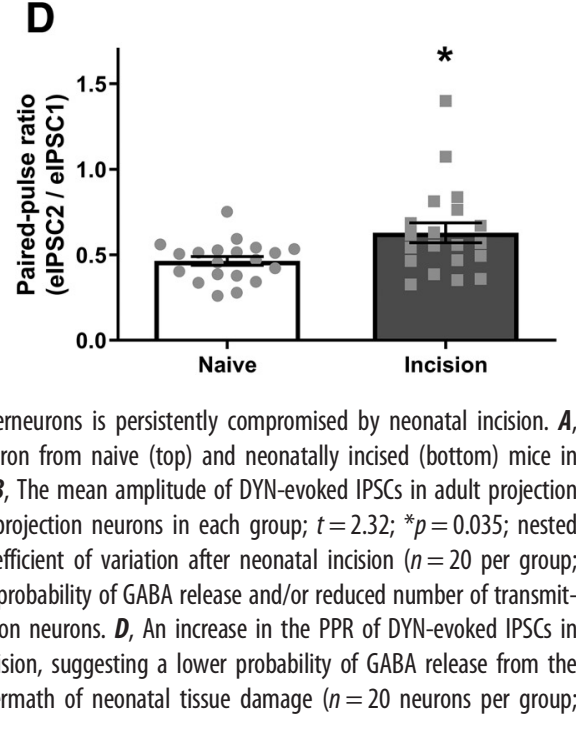


suggest that neonatal tissue damage reduces the activation of mature DYN neurons in response to sensory input, likely affecting the ability of these interneurons to inhibit downstream somatosensory circuits in a feedforward manner.

\section{DYN inhibitory synapses onto} projection neurons are persistently weakened by neonatal injury

After finding long-term deficits in the primary afferent-evoked output of DYN interneurons following neonatal tissue injury, we next sought to determine whether critical downstream targets of DYN inhibitory neurons were also affected. Identifying potential alterations in the modulation of lamina I projection neurons is critical, given the importance of these neurons for somatosensation and persistent pain (Kauppila, 1997; Mantyh et al., 1997; Nichols et al., 1999). DYN interneurons form inhibitory synapses, which are primarily GABAergic, directly onto projection neurons (C.L.B., L. Styczynski, E.K.S., M.L.B., unpublished observations; Hachisuka et al., 2020), yet it is unknown whether this key synaptic connection is affected by peripheral tissue damage at any stage of development. Therefore, we investigated the functional properties of DYN inhibitory synapses onto adult projection neurons after surgical injury sustained during the neonatal period.

Spinal DYN interneurons expressing ChR2 were activated via optogenetic stimulation to evoke monosynaptic IPSCs in laminaI spinoparabrachial neurons (see Materials and Methods). Importantly, the mean amplitude of DYN-evoked IPSCs in adult projection neurons was significantly decreased by neonatal incision compared with naive littermate controls $(n=20$ projection neurons in each group; $t=2.32 ; p=$ 0.035 ; nested unpaired $t$ test; Fig. $6 A$, $B)$. Additionally, a higher coefficient of variation was observed after neonatal incision ( $n=20$ neurons in each group; $t=2.77 ; p=0.014$; nested unpaired $t$ test; Fig. $6 C$ ), which suggests a persistent reduction in either the probability of GABA release or the number of neurotransmitter release sites at DYN synapses onto mature projection neurons after early-life injury. The PPR was also significantly higher in the neonatally incised mice $(n=20$ neurons in each group; $t=2.34 ; p=0.034$; nested unpaired $t$ test;

A

B
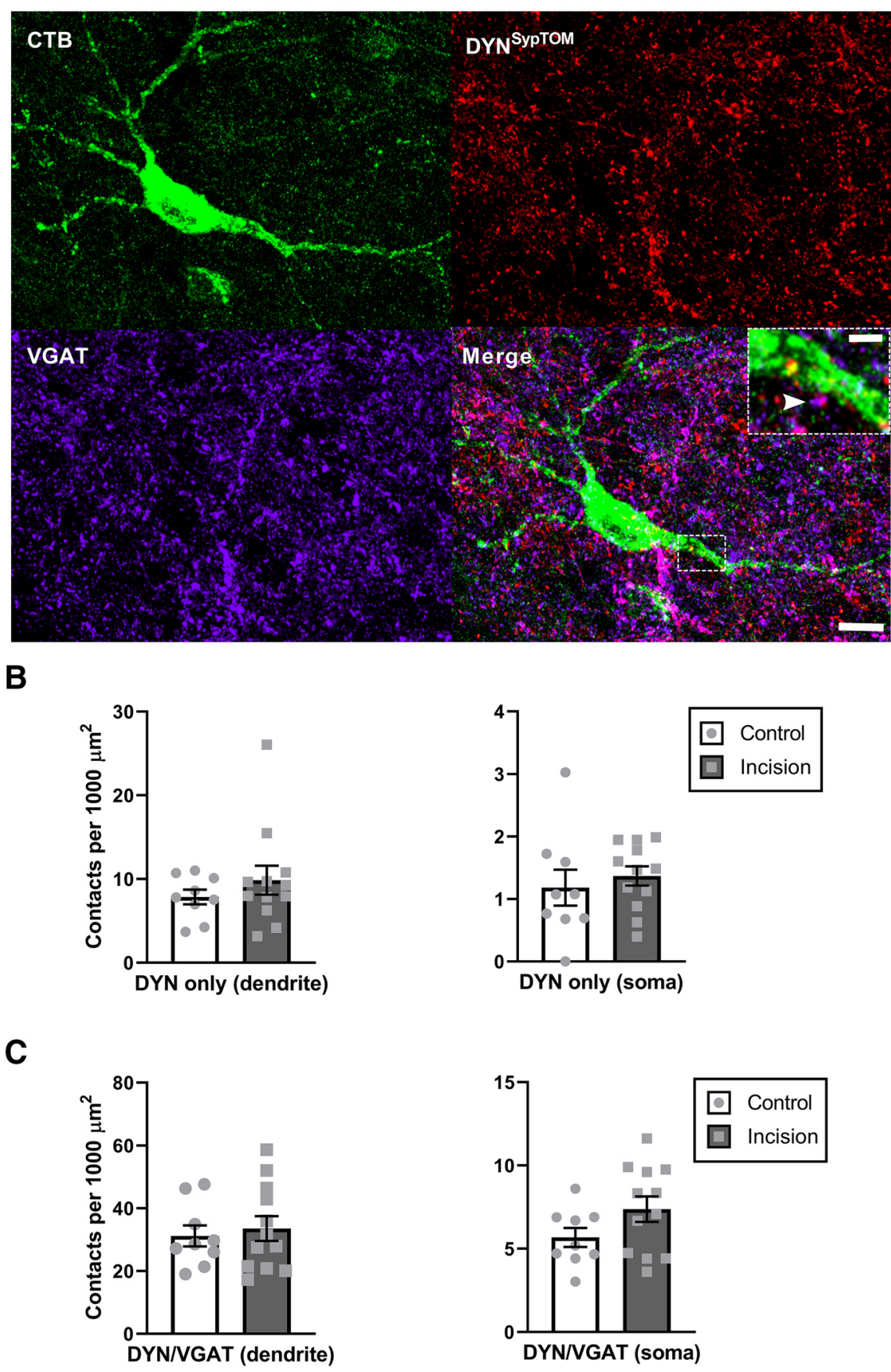

Figure 7. Early-life injury does not alter the innervation of adult projection neurons by inhibitory DYN neurons. $\boldsymbol{A}$, Representative images of (TB-labeled lamina I projection neuron (top left), synaptophysin-tdTOM labeling of DYN presynaptic terminals (DYN ${ }^{\text {SypTOM}}$; top right), VGAT-labeled inhibitory boutons (bottom left), and the merged image (bottom right). Scale bar, $20 \mu \mathrm{m}$. Inset (in the merged image), Magnification of an inhibitory DYN bouton (arrowhead), as defined by the coexpression of VGAT (purple) and DYN ${ }^{\text {SypTOM }}$ (red), in apposition to the dendrite of an identified spinoparabrachial neuron (green). Scale bar, $5 \mu \mathrm{m}$. $\boldsymbol{B}$, Neonatal injury had no significant effect on putative excitatory DYN (i.e., synaptophysin-tdTOM only) contacts onto projection neuron dendrites (naive: $n=9$ neurons; incision: $n=12 ; t=0.57 ; p=0.60$; nested unpaired $t$ test; left) or somata ( $t=0.62 ; p=0.54$, nested unpaired $t$ test; right). $C$, P3 incision also failed to significantly influence the density of inhibitory DYN (i.e., synaptophysin-tdTOM/VGAT) terminals in apposition to the dendrites $(t=0.41 ; p=0.70$; nested unpaired $t$ test; left) or somata ( $t=1.3 ; p=0.25$; nested unpaired $t$ test; right) of adult projection neurons (naive: $n=9$; incision: $n=12$ ).

Fig. 6D), thereby supporting a reduced probability of GABA release from inhibitory DYN synapses in the adult SDH after early injury.

It is possible that a disruption of the normal innervation of projection neurons by spinal DYN neurons also contributes to 
A

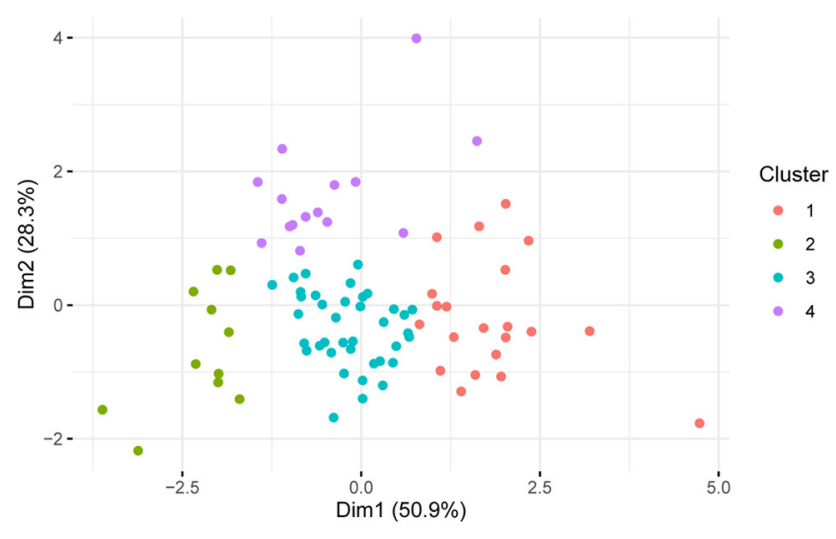

C

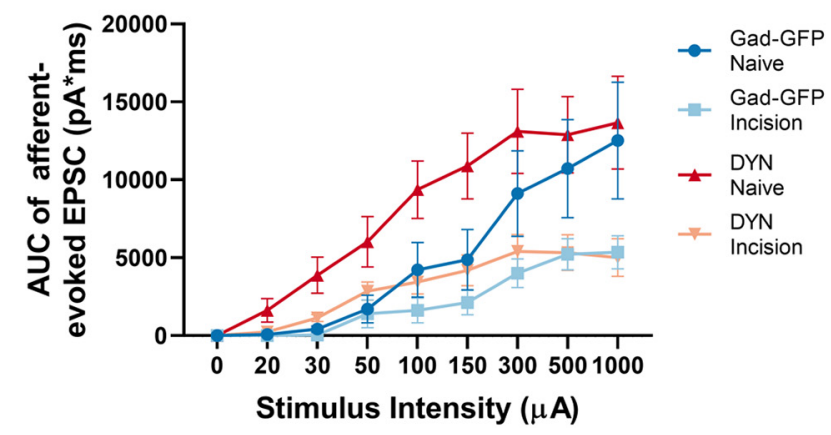

B

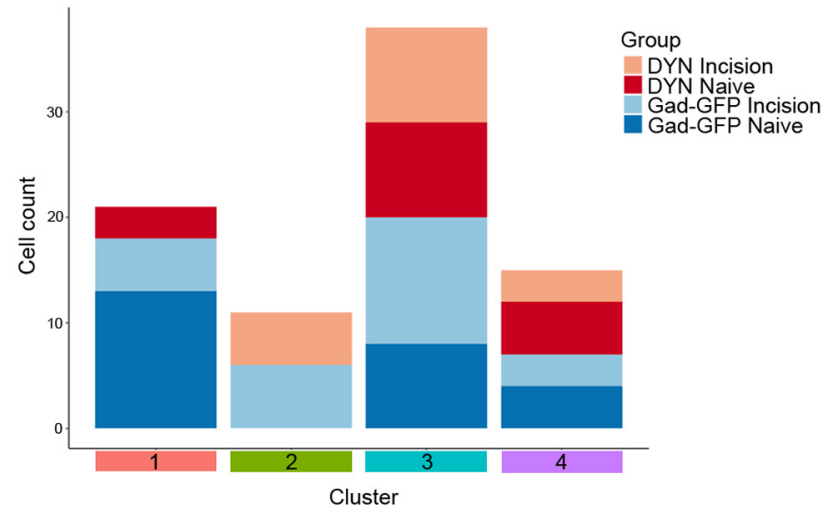

D

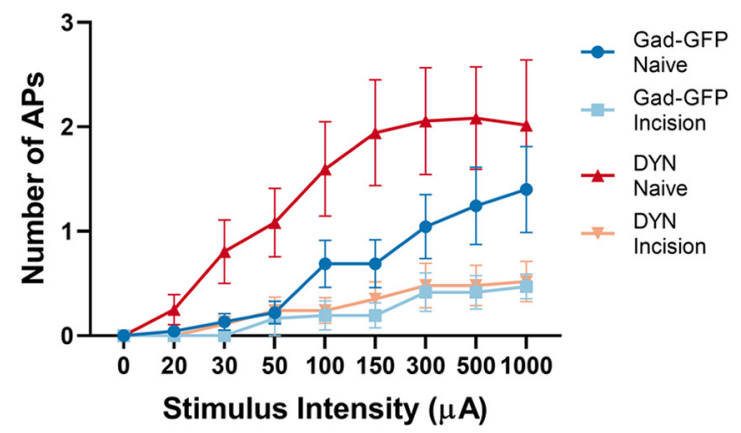

Figure 8. Comparison of the effects of neonatal injury on the electrophysiological properties of DYN versus Gad67-GFP interneurons in the adult SDH. A, Plot of principal components analysis (Dim1, Dimension 1; Dim2, Dimension 2) showing the unbiased distribution of mature DYN neurons and Gad-GFP neurons into four clusters based on $k$-means clustering, where each point represents an individual neuron. $\boldsymbol{B}$, Histogram of the number of SDH cells in each cluster, where each neuron belonged to one of four experimental groups: adult DYN neurons from P3-incised mice (DYN Incision; light pink), adult DYN neurons from naive mice (DYN Naive; red), adult Gad67-GFP neurons from P3-incised mice (Gad-GFP Incision; light blue), and adult Gad67-GFP neurons from naive mice (Gad-GFP Naive; dark blue). Cluster 2 is composed entirely of neurons from neonatally injured mice, although DYN and Gad-GFP neurons are represented in roughly equal numbers within this cluster. Furthermore, there was no absolute segregation of DYN and GABAergic neurons in any of the four clusters. C, Overall glutamatergic drive onto mature DYN neurons from naive (red) and P3-incised (light pink) mice (data replotted from Fig. 3) was compared with that observed onto Gad67-GFP neurons from naive ( $n=15$; dark blue) and P3-incised $\left(n=12\right.$; light blue) mice (data replotted from Li and Baccei (2019)). There was no significant interaction between injury and cell type $\left(F_{(1,65)}=0.674 ; p=0.415\right.$; mixed-effects model). $\boldsymbol{D}$, Similarly, there was no significant interaction between injury and cell type $\left(F_{(1,65)}=1.550 ; p=0.218\right.$; mixed-effects model) in terms of the effect of $\mathrm{P} 3$ incision on primary afferent-evoked firing.

the above decrease in the strength of DYN inhibitory synapses onto lamina I spinoparabrachial neurons after neonatal injury. To address this issue, we estimated the number of DYN presynaptic boutons that were apposed to adult spinoparabrachial neurons from naive and neonatally incised mice. DYN presynaptic terminals were genetically identified via the expression of a Credependent synaptophysin-tdTOM fusion protein, whereas inhibitory terminals were labeled via immunohistochemistry using a primary antibody targeting VGAT (Fig. 7A). Importantly, we found no significant effect of $\mathrm{P} 3$ incision on the density of inhibitory DYN boutons (i.e., expressing both synaptophysin-tdTOM and VGAT; Fig. 7A) in apposition to the dendrites (naive: $n=9$ neurons; incision: $n=12$; $t=0.41 ; p=0.70$; nested unpaired $t$ test; Fig. 7C, left) or somata $(t=1.3 ; p=0.25$; nested unpaired $t$ test; Fig. $7 C$, right) of mature projection neurons. Additionally, neonatal injury did not affect the density of presumed excitatory DYN inputs (i.e., expressing synaptophysin-tdTOM but not VGAT; Fig. $7 A$ ) onto the dendrites (naive: $n=9$; incision: $n=12$; $t=0.57 ; p=0.60$; nested unpaired $t$ test; Fig. $7 B$, left) or cell bodies $(t=0.62 ; p=0.54$; nested unpaired $t$ test; Fig. $7 B$, right $)$ of adult spinoparabrachial neurons. Although a rigorous assessment of synaptic density would require analysis at the ultrastructural level, these results argue against the notion that P3 injury significantly alters the structural innervation of mature projection neurons by inhibitory DYN interneurons, thereby suggesting that the observed reduction in the DYN-evoked IPSC amplitude (Fig. $6 B$ ) is most likely explained by a lower probability of GABA release.

Does neonatal injury selectively affect the DYN subpopulation of GABAergic neurons in the mature SDH? A key unanswered question is whether the long-term changes in the intrinsic membrane properties and synaptic connectivity of inhibitory SDH interneurons are restricted to those cells derived from the DYN lineage, or rather occur across the wider GABAergic population. As a first step toward addressing this issue, we capitalized on the data collected during our previous studies, which investigated the effects of $\mathrm{P} 3$ hindpaw incision on the intrinsic excitability of laminae I-II GABAergic neurons identified via the expression of Gad67-EGFP (Li and Baccei, 2014). Data on membrane capacitance, resting membrane potential, membrane resistance, and rheobase from those Gad-GFP neurons were pooled with the same measures obtained from the current recordings from DYN-tdTOM neurons (Fig. 2) and 
subjected to unbiased principal components analysis and $k$ means clustering. Silhouette analysis revealed an optimal number of four clusters (Fig. 8A). As expected given that these same variables were used for $k$-means clustering, significant differences in membrane capacitance, resting membrane potential, membrane resistance, and rheobase ( $p<0.0001$; Kruskal-Wallis test) were observed across clusters. More importantly, while Cluster 2 was composed only of SDH neurons derived from neonatally incised mice, DYN neurons and GABAergic neurons were represented fairly equally within this cluster (Fig. $8 B$ ). In addition, there was no absolute segregation of DYN and GABAergic neurons in any of the four clusters (Fig. 8B).

Furthermore, if the persistent effects of P3 incision were indeed unique to DYN interneurons within the mature $\mathrm{SDH}$, one might predict that the injury-evoked changes would be more dramatic when selectively sampling DYN-tdTOM neurons compared with recording from the wider GABAergic population, given that DYN neurons only represent $\sim 30 \%$ of all inhibitory interneurons within laminae I-II (as identified via colocalization with Pax2) (Boyle et al., 2017). However, a statistical comparison of the effects of neonatal incision on primary afferent-evoked glutamatergic drive to adult DYN neurons (data replotted from Fig. 3C) to those recently documented for adult Gad67-GFP neurons ( $\mathrm{Li}$ and Baccei, 2019) revealed a significant overall effect of injury $\left(F_{(1,65)}=8.08 ; p=0.006\right.$; mixed-effects model $)$ but no effect of cell type $\left(F_{(1,65)}=2.205 ; p=0.142\right)$ and no significant interaction between injury and cell type $\left(F_{(1,65)}=0.674\right.$; $p=0.415$; Fig. $8 C$ ). In addition, the influence of $\mathrm{P} 3$ incision on afferent-evoked AP discharge was similar in the DYN and Gad67-GFP populations, as we observed a significant effect of injury $\left(F_{(1,65)}=7.642 ; p=0.007\right.$; mixed-effects model $)$ but no effect of cell type $\left(F_{(1,65)}=2.199 ; p=0.143\right)$ and no significant interaction between injury and cell type $\left(F_{(1,65)}=1.550\right.$; $p=0.218$; Fig. $8 D$ ).

Although it is clear that further studies are needed to rigorously address this issue, the available results collectively suggest that the effects of early-life tissue injury on the electrophysiological properties of DYN neurons are not fundamentally distinct from those seen across the larger GABAergic population within the SDH.

\section{Discussion}

These results identify functional deficits in a genetically defined subpopulation of interneurons within the adult mouse $\mathrm{SDH}$ following neonatal tissue damage. Surgical injury at P3 weakened sensory afferent drive onto mature interneurons derived from the prodynorphin lineage, dampened their intrinsic excitability, and decreased the efficacy of their inhibitory synapses onto lamina I spinoparabrachial neurons by lowering the probability of GABA release. Collectively, these data yield new insight into the potential circuit mechanisms by which earlylife injury can persistently enhance the excitability of pain pathways in the adult CNS.

Neonatal tissue damage in rodents evokes two long-term changes in pain sensitivity that are temporally and spatially distinct. A global reduction in baseline pain sensitivity is observed, which does not emerge until adolescence (Ren et al., 2004; LaPrairie and Murphy, 2007) and may reflect stronger descending inhibition following neonatal injury (Zhang et al., 2010; Walker et al., 2015). The delayed onset of this hypoalgesia could be explained by the slow maturation of descending inhibitory pathways during early life, as descending facilitation dominates throughout the first three postnatal weeks (Fitzgerald and Koltzenburg, 1986; Hathway et al., 2009). Meanwhile, in the same animals, an exacerbated degree of pain hypersensitivity is seen following repeat injury (termed "priming"), which is evident immediately after the resolution of the initial injury and appears more localized, as priming was not observed if the subsequent injury occurred in the contralateral hindpaw (Ren et al., 2004; Moriarty et al., 2019). Recent work indicates that priming involves the sensitization of central nociceptive circuits and does not require changes occurring at the peripheral site of injury. First, electrical stimulation of the tibial nerve (as a substitute for adult injury) can also precipitate priming following neonatal hindpaw incision (Beggs et al., 2012). In addition, adult mice which sustained hindpaw injury as neonates exhibit enhanced mechanical allodynia following incision of the ipsilateral forepaw during adulthood (Moriarty et al., 2019). Therefore, it is possible that neonatal tissue damage leads to a persistent sensitization of ipsilateral spinal nociceptive networks that initially remains latent due to increased descending inhibition from the brainstem, but is later unmasked by subsequent reinjury.

It is becoming clear that neonatal injury evokes widespread disruptions in inhibitory synaptic transmission in the mature $\mathrm{SDH}$, which can undoubtedly sensitize spinal pain networks (Yaksh, 1989; Sivilotti and Woolf, 1994; Foster et al., 2015). For example, neonatal incision reduced both the GABAergic and glycinergic components of primary afferent-evoked FFI onto lamina I spinoparabrachial neurons, which appears mediated by changes in the functional properties of SDH circuits rather than an overt loss of inhibitory inputs to projection neurons (Li et al., 2015). Such a loss of FFI would be expected to potentiate ascending nociceptive transmission to the brain and thereby enhance the susceptibility to developing persistent pain (Mantyh et al., 1997; Nichols et al., 1999; Huang et al., 2019). Given their high prevalence in the DH (Boyle et al., 2017), direct innervation by primary afferents (Fig. 3), and ability to directly inhibit lamina I projection neurons (Fig. 6) (Hachisuka et al., 2020), it seems highly likely that spinal DYN-lineage neurons represent a critical component of this feedforward inhibitory pathway linking primary sensory neurons to projection neurons.

Importantly, the present results demonstrate that this putative feedforward pathway is significantly compromised by neonatal tissue damage via multiple mechanisms operating in parallel. First, a disruption in the normal balance of primary afferent-evoked excitation versus inhibition onto mature DYN interneurons (Figs. 3, 4), combined with a reduction in their intrinsic membrane excitability (Fig. 2), results in a marked decrease in afferent-evoked firing within this population (Fig. 5). Furthermore, DYN synapses onto lamina I spinoparabrachial neurons are persistently weakened by neonatal surgical injury, secondary to a long-term reduction in the probability of GABA release (Fig. 6). It will ultimately be essential to elucidate the somatotopy governing these injuryevoked alterations in the function of spinal DYN circuits. Little is known about the spatial distribution of the deficits in synaptic inhibition following early-life injury, although the reduction in spontaneous glycinergic transmission after P3 hindpaw incision does not extend to the contralateral SDH (Li et al., 2013). Furthermore, it remains to be determined whether a discrete critical period exists for these injuryevoked changes in spinal DYN networks. Notably, the persistent changes in spontaneous inhibitory signaling ( $\mathrm{Li}$ et al., 
2013), spike timing-dependent LTP (Li and Baccei, 2016), and pain behaviors (Ren et al., 2004; Walker et al., 2009) seen following neonatal tissue damage are not observed following similar injuries that occur after the first postnatal week. Nonetheless, under certain circumstances, mature spinal nociceptive circuits can clearly be sensitized by injuries sustained during adulthood via a well-documented process termed "hyperalgesic priming" (Joseph et al., 2003; Ferrari et al., 2015; Megat et al., 2018). Unfortunately, the degree to which the mechanisms underlying hyperalgesic priming and neonatal priming overlap remains to be elucidated.

Early surgical injury dampens sensory afferent drive to the wider population of Gad67-GFP interneurons within the adult SDH (Li and Baccei, 2019) to a similar degree as observed in mature DYN neurons (Fig. 8C,D). Given this observation, along with the lack of separation between DYN and Gad67-GFP neurons in our cluster analysis of intrinsic membrane properties under normal and pathologic conditions (Fig. 8B), it seems highly likely that neonatal injury modulates the function of several subtypes of GABAergic neurons across the mature SDH. Nonetheless, it will clearly be important for future studies to consider how early-life injury affects other major classes of inhibitory interneurons (Polgár et al., 2011; Sardella et al., 2011; Tiong et al., 2011). For example, it would be of great interest to characterize the effects of $\mathrm{P} 3$ injury on parvalbumin interneurons given their prevalence in the SDH (Boyle et al., 2017) and their role in suppressing mechanical pain (Petitjean et al., 2015), in light of the exacerbated mechanical allodynia that characterizes neonatal priming (Ren et al., 2004; Moriarty et al., 2019).

It should be noted that our electrophysiological recordings sampled both inhibitory and excitatory DYN-lineage neurons (Duan et al., 2014; Boyle et al., 2017). Intersectional genetic strategies (Duan et al., 2014; Bourane et al., 2015) could be used to distinguish the effects of P3 incision on the excitability of GABAergic versus glutamatergic DYN neurons within the adult $\mathrm{SDH}$. Interestingly, mature laminae I-II neurons that lacked Gad67-GFP expression, the vast majority ( 80\%-90\%) of which correspond to glutamatergic interneurons (Dougherty et al., 2009), exhibited similar responses to neonatal injury as adjacent GABAergic neurons ( $\mathrm{Li}$ and Baccei, 2019). This raises the possibility that while neonatal tissue damage evokes cell type-dependent effects within the adult SDH, the major difference lies in the response of interneurons versus projection neurons to the injury rather than a distinct modulation of excitatory versus inhibitory neurons. In this regard, the influence of neonatal injury on the efficacy of DYN synapses onto other interneurons within the mature DH should also be examined in future studies. Another limitation of the current study is that it exclusively sampled DYN neurons in the superficial laminae, while DYN neurons are also located in the deeper DH (Duan et al., 2014) and could exhibit distinct responses to early-life injury. Interestingly, since superficial DYN neurons derived from the B5-I lineage are important for the regulation of itch (Kardon et al., 2014), the observed long-term deficits in DYN neuron-mediated inhibition after neonatal injury could predict altered sensitivity to pruritic stimuli during adulthood. However, it remains unclear whether neonatal tissue damage can persistently modulate itch processing throughout life.

The molecular mechanisms underlying these persistent deficits in spinal DYN circuits following neonatal injury remain to be identified. The documented effects on both the intrinsic membrane excitability (Fig. 2) and probability of presynaptic GABA release (Fig. 6) are consistent with complex, multifaceted changes in the functional properties of adult spinal DYN neurons after noxious stimulation during early life. Recent advances in the genetic profiling of identified subpopulations of spinal neurons (Chamessian et al., 2018; Serafin et al., 2019) would allow for the future investigation into how neonatal injury influences gene expression in DYN interneurons within the mature $\mathrm{DH}$. However, post-transcriptional regulation could also be critical for the persistent, injury-evoked alterations in spinal DYN circuits. Notably, the complement of mRNAs that are actively translated are not always well predicted by the overall pool of mRNAs detected by RNAseq (Ingolia, 2016; Fortelny et al., 2017). Unfortunately, while the translating ribosome affinity purification approach (Heiman et al., 2008) has been successfully applied to the dorsal root ganglia (Megat et al., 2019a,b), the degree to which it can be used to elucidate the translatome of identified subpopulations of DH neurons remains to be determined.

In conclusion, the present findings represent the first step toward elucidating the long-term effects of neonatal injury on the function of identified inhibitory microcircuits within the spinal $\mathrm{DH}$. Such information may yield new mechanistic insight into the synaptic pathways which must ultimately be restored to prevent an increased susceptibility to chronic pain following tissue damage during early life.

\section{References}

Beggs S, Currie G, Salter MW, Fitzgerald M, Walker SM (2012) Priming of adult pain responses by neonatal pain experience: maintenance by central neuroimmune activity. Brain 135:404-417.

Bourane S, Duan B, Koch SC, Dalet A, Britz O, Garcia-Campmany L, Kim E, Cheng L, Ghosh A, Ma Q, Goulding M (2015) Gate control of mechanical itch by a subpopulation of spinal cord interneurons. Science 350:550554.

Boyle KA, Gutierrez-Mecinas M, Polgár E, Mooney N, O’Connor E, Furuta T, Watanabe M, Todd AJ (2017) A quantitative study of neurochemically defined populations of inhibitory interneurons in the superficial dorsal horn of the mouse spinal cord. Neuroscience 363:120133.

Brennan TJ, Vandermeulen EP, Gebhart GF (1996) Characterization of a rat model of incisional pain. Pain 64:493-501.

Cameron D, Polgár E, Gutierrez-Mecinas M, Gomez-Lima M, Watanabe M, Todd AJ (2015) The organisation of spinoparabrachial neurons in the mouse. Pain 156:2061-2071.

Chamessian A, Young M, Qadri Y, Berta T, Ji RR, Van de Ven T (2018) Transcriptional profiling of somatostatin interneurons in the spinal dorsal horn. Sci Rep 8:6809.

Cheng L, Arata A, Mizuguchi R, Qian Y, Karunaratne A, Gray PA, Arata S, Shirasawa S, Bouchard M, Luo P, Chen CL, Busslinger M, Goulding M, Onimaru H, Ma Q (2004) Tlx3 and Tlx1 are post-mitotic selector genes determining glutamatergic over GABAergic cell fates. Nat Neurosci 7:510-517.

Dougherty KJ, Sawchuk MA, Hochman S (2009) Phenotypic diversity and expression of GABAergic inhibitory interneurons during postnatal development in lumbar spinal cord of glutamic acid decarboxylase 67-green fluorescent protein mice. Neuroscience 163:909-919.

Duan B, Cheng L, Bourane S, Britz O, Padilla C, Garcia-Campmany L, Krashes M, Knowlton W, Velasquez T, Ren X, Ross SE, Lowell BB, Wang Y, Goulding M, Ma Q (2014) Identification of spinal circuits transmitting and gating mechanical pain. Cell 159:1417-1432.

Ferrari LF, Araldi D, Levine JD (2015) Distinct terminal and cell body mechanisms in the nociceptor mediate hyperalgesic priming. J Neurosci 35:6107-6116.

Fitzgerald M (2005) The development of nociceptive circuits. Nat Rev Neurosci 6:507-520. 
Fitzgerald M, Koltzenburg M (1986) The functional development of descending inhibitory pathways in the dorsolateral funiculus of the newborn rat spinal cord. Dev Brain Res 24:261-270.

Fortelny N, Overall CM, Pavlidis P, Freue GV (2017) Can we predict protein from mRNA levels? Nature 547:E19-E20.

Foster E, Wildner H, Tudeau L, Haueter S, Ralvenius WT, Jegen M, Johannssen H, Hösli L, Haenraets K, Ghanem A, Conzelmann KK, Bosl M, Zeilhofer HU (2015) Targeted ablation, silencing, and activation establish glycinergic dorsal horn neurons as key components of a spinal gate for pain and itch. Neuron 85:1289-1304.

Hachisuka J, Koerber HR, Ross SE (2020) Selective-cold output via a distinct subset of lamina I spinoparabrachial neurons. Pain 161:185-194.

Harrison W, Goodman D (2015) Epidemiologic trends in neonatal intensive care, 2007-2012. JAMA Pediatr 169:855-862.

Hathway G, Koch S, Low L, Fitzgerald M (2009) The changing balance of brainstem-spinal cord modulation of pain processing over the first weeks of rat postnatal life. J Physiol 587:2927-2935.

Heiman M, Schaefer A, Gong S, Peterson JD, Day M, Ramsey KE, SuárezFariñas M, Schwarz C, Stephan DA, Surmeier DJ, Greengard P, Heintz N (2008) A translational profiling approach for the molecular characterization of CNS cell types. Cell 135:738-748.

Hermann C, Hohmeister J, Demirakça S, Zohsel K, Flor H (2006) Long-term alteration of pain sensitivity in school-aged children with early pain experiences. Pain 125:278-285.

Hohmeister J, Kroll A, Wollgarten-Hadamek I, Zohsel K, Demirakça S, Flor H, Hermann C (2010) Cerebral processing of pain in school-aged children with neonatal nociceptive input: an exploratory fMRI study. Pain 150:257-267.

Huang T, Lin SH, Malewicz NM, Zhang Y, Zhang Y, Goulding M, LaMotte RH, Ma Q (2019) Identifying the pathways required for coping behaviours associated with sustained pain. Nature 565:86-90.

Huang J, Polgár E, Solinski HJ, Mishra SK, Tseng PY, Iwagaki N, Boyle KA, Dickie AC, Kriegbaum MC, Wildner H, Zeilhofer HU, Watanabe M, Riddell JS, Todd AJ, Hoon MA (2018) Circuit dissection of the role of somatostatin in itch and pain. Nat Neurosci 21:707-716.

Ingolia NT (2016) Ribosome footprint profiling of translation throughout the genome. Cell 165:22-33.

Joseph EK, Parada CA, Levine JD (2003) Hyperalgesic priming in the rat demonstrates marked sexual dimorphism. Pain 105:143-150.

Kardon AP, Polgár E, Hachisuka J, Snyder LM, Cameron D, Savage S, Cai X, Karnup S, Fan CR, Hemenway GM, Bernard CS, Schwartz ES, Nagase H, Schwarzer C, Watanabe M, Furuta T, Kaneko T, Koerber HR, Todd AJ, Ross SE (2014) Dynorphin acts as a neuromodulator to inhibit itch in the dorsal horn of the spinal cord. Neuron 82:573-586.

Kassambara A, Mundt F (2019) factoextra: extract and visualize the results of multivariate data analyses. In: R package version 1.0.6.

Kauppila T (1997) Spinalization increases the mechanical stimulationinduced withdrawal reflex threshold after a sciatic cut in the rat. Brain Res 770:310-312.

LaPrairie JL, Murphy AZ (2007) Female rats are more vulnerable to the longterm consequences of neonatal inflammatory injury. Pain 132:S124S133.

Larsson M (2017) Pax2 is persistently expressed by GABAergic neurons throughout the adult rat dorsal horn. Neurosci Lett 638:96-101.

LeResche L (1999) Gender considerations in the epidemiology of chronic pain. Epidemiol Pain 17:43-52.

Li J, Baccei ML (2014) Neonatal tissue injury reduces the intrinsic excitability of adult mouse superficial dorsal horn neurons. Neuroscience 256:392402.

Li J, Baccei ML (2016) Neonatal tissue damage promotes spike timing-dependent synaptic long-term potentiation in adult spinal projection neurons. J Neurosci 36:5405-5416.

Li J, Baccei ML (2019) Neonatal injury alters sensory input and synaptic plasticity in GABAergic interneurons of the adult mouse dorsal horn. J Neurosci 39:7815-7825.

Li J, Blankenship ML, Baccei ML (2013) Deficits in glycinergic inhibition within adult spinal nociceptive circuits after neonatal tissue damage. Pain 154:1129-1139.

Li J, Kritzer E, Craig PE, Baccei ML (2015) Aberrant synaptic integration in adult lamina I projection neurons following neonatal tissue damage. J Neurosci 35:2438-2451.
Maechler M, Rousseeuw P, Struyf A, Hubert M, Hornik K (2018) cluster: cluster analysis basics and extensions. In: R package version 2.0.7-1.

Mantyh PW, Rogers SD, Honore P, Allen BJ, Ghilardi JR, Li J, Daughters RS, Lappi DA, Wiley RG, Simone DA (1997) Inhibition of hyperalgesia by ablation of lamina I spinal neurons expressing the substance P receptor. Science 278:275-279.

Megat S, Shiers S, Moy JK, Barragan-Iglesias P, Pradhan G, Seal RP, Dussor G, Price TJ (2018) A critical role for dopamine D5 receptors in pain chronicity in male mice. J Neurosci 38:379-397.

Megat S, Ray PR, Tavares-Ferreira D, Moy JK, Sankaranarayanan I, Wanghzou A, Lou TF, Barragan-Iglesias P, Campbell ZT, Dussor G, Price TJ (2019a) Differences between dorsal root and trigeminal ganglion nociceptors in mice revealed by translational profiling. J Neurosci 39:6829-6847.

Megat S, Ray PR, Moy JK, Lou TF, Barragán-Iglesias P, Li Y, Pradhan G, Wanghzou A, Ahmad A, Burton MD, North RY, Dougherty PM, Khoutorsky A, Sonenberg N, Webster KR, Dussor G, Campbell ZT, Price TJ (2019b) Nociceptor translational profiling reveals the Ragulator-Rag GTPase complex as a critical generator of neuropathic pain. J Neurosci 39:393-411.

Melzack R, Wall PD (1965) Pain mechanisms: a new theory. Science 150:971-979.

Moriarty O, Harrington L, Beggs S, Walker S (2018) Opioid analgesia and the somatosensory memory of neonatal surgical injury in the adult rat. $\mathrm{Br}$ J Anaesth 121:314-324.

Moriarty O, Tu Y, Sengar AS, Salter MW, Beggs S, Walker SM (2019) Priming of adult incision response by early life injury: neonatal microglial inhibition has persistent but sexually dimorphic effects in adult rats. J Neurosci 39:3081-3093.

Nichols ML, Allen BJ, Rogers SD, Ghilardi JR, Honore P, Luger NM, Finke MP, Li J, Lappi DA, Simone DA, Mantyh PW (1999) Transmission of chronic nociception by spinal neurons expressing the substance $\mathrm{P}$ receptor. Science 286:1558-1561.

Paxinos G, Franklin KB (2012) The mouse brain in stereotaxic coordinates. San Diego: Academic.

Petitjean H, Pawlowski SA, Fraine SL, Sharif B, Hamad D, Fatima T, Berg J, Brown CM, Jan LY, Ribeiro-da-Silva A, Braz JM, Basbaum AI, Sharif-Naeini R (2015) Dorsal horn parvalbumin neurons are gatekeepers of touch-evoked pain after nerve injury. Cell Rep 13:12461257.

Pfeffer CK, Xue M, He M, Huang ZJ, Scanziani M (2013) Inhibition of inhibition in visual cortex: the logic of connections between molecularly distinct interneurons. Nat Neurosci 16:1068-1076.

Polgár E, Sardella TC, Watanabe M, Todd AJ (2011) Quantitative study of NPY-expressing GABAergic neurons and axons in rat spinal dorsal horn. J Comp Neurol 519:1007-1023.

Ren K, Anseloni V, Zou SP, Wade E, Novikova S, Ennis M, Traub R, Gold M, Dubner R, Lidow M (2004) Characterization of basal and re-inflammation-associated long-term alteration in pain responsivity following short-lasting neonatal local inflammatory insult. Pain 110:588-596.

Rousseeuw PJ (1987) Silhouettes: a graphical aid to the interpretation and validation of cluster analysis. J Comput Appl Math 20:53-65.

Roux L, Buzsáki G (2015) Tasks for inhibitory interneurons in intact brain circuits. Neuropharmacology 88:10-23.

Sardella TC, Polgár E, Garzillo F, Furuta T, Kaneko T, Watanabe M, Todd AJ (2011) Dynorphin is expressed primarily by GABAergic neurons that contain galanin in the rat dorsal horn. Mol Pain 7:76.

Serafin EK, Chamessian A, Li J, Zhang X, McGann A, Brewer CL, Berta T, Baccei M (2019) Transcriptional profile of spinal dynorphin-lineage interneurons in the developing mouse. Pain 160:2380-2397.

Simons SH, van Dijk M, Anand KS, Roofthooft D, van Lingen RA, Tibboel D (2003) Do we still hurt newborn babies? A prospective study of procedural pain and analgesia in neonates. Arch Pediatr Adolesc Med 157:1058-1064.

Sivilotti L, Woolf CJ (1994) The contribution of GABAA and glycine receptors to central sensitization: disinhibition and touch-evoked allodynia in the spinal cord. J Neurophysiol 72:169-179.

Ting JT, Daigle TL, Chen Q, Feng G (2014) Acute brain slice methods for adult and aging animals: application of targeted patch clamp analysis and optogenetics. In: Patch-clamp methods and protocols, pp 221-242. New York: Springer. 
Tiong SY, Polgár E, van Kralingen JC, Watanabe M, Todd AJ (2011) Galanin-immunoreactivity identifies a distinct population of inhibitory interneurons in laminae I-III of the rat spinal cord. Mol Pain 7:36.

Todd AJ (2010) Neuronal circuitry for pain processing in the dorsal horn. Nat Rev Neurosci 11:823-836.

Todd AJ, Puskár Z, Spike RC, Hughes C, Watt C, Forrest L (2002) Projection neurons in lamina I of rat spinal cord with the neurokinin 1 receptor are selectively innervated by substance P-containing afferents and respond to noxious stimulation. J Neurosci 22:4103-4113.

Torsney C, MacDermott AB (2006) Disinhibition opens the gate to pathological pain signaling in superficial neurokinin 1 receptor-expressing neurons in rat spinal cord. J Neurosci 26:1833-1843.

Walker SM, Tochiki KK, Fitzgerald M (2009) Hindpaw incision in early life increases the hyperalgesic response to repeat surgical injury: critical period and dependence on initial afferent activity. Pain 147:99-106.
Walker SM, Fitzgerald M, Hathway GJ (2015) Surgical injury in the neonatal rat alters the adult pattern of descending modulation from the rostroventral medulla. Anesthesiology 122:1391-1400.

Walker SM, Beggs S, Baccei ML (2016) Persistent changes in peripheral and spinal nociceptive processing after early tissue injury. Exp Neurol 275: 253-260.

Xu H, Jeong HY, Tremblay R, Rudy B (2013) Neocortical somatostatinexpressing GABAergic interneurons disinhibit the thalamorecipient layer 4. Neuron 77:155-167.

Yaksh TL (1989) Behavioral and autonomic correlates of the tactile evoked allodynia produced by spinal glycine inhibition: effects of modulatory receptor systems and excitatory amino acid antagonists. Pain 37:111-123.

Zhang YH, Wang XM, Ennis M (2010) Effects of neonatal inflammation on descending modulation from the rostroventromedial medulla. Brain Res Bull 83:16-22. 